\title{
ON THE EXISTENCE OF SOLUTIONS FOR THE NONSTATIONARY STOKES SYSTEM WITH SLIP BOUNDARY CONDITIONS IN GENERAL SOBOLEV-SLOBODETSKII AND BESOV SPACES
}

\author{
WISAM ALAME \\ Technical University of Warsaw \\ Pl. Politechniki 1, 00-661 Warszawa, Poland \\ E-mail: w.alame@wp.pl
}

\begin{abstract}
We prove the existence of solutions to the evolutionary Stokes system in a bounded domain $\Omega \subset \mathbb{R}^{3}$. The main result shows that the velocity belongs either to $W_{p}^{2 s+2, s+1}\left(\Omega^{T}\right)$ or to $B_{p, q}^{2 s+2, s+1}\left(\Omega^{T}\right)$ with $p>3$ and $s \in \mathbb{R}_{+} \cup\{0\}$. The proof is divided into two steps. First the existence in $W_{p}^{2 k+2, k+1}$ for $k \in \mathbb{N}$ is proved. Next applying interpolation theory the existence in Besov spaces in a half space is shown. Finally the technique of regularizers implies the existence in a bounded domain. The result is generalized to the spaces $W_{p}^{2 s, s}\left(\Omega^{T}\right)$ and $B_{p, q}^{2 s, s}$ with $p>2$ and $s \in(1 / 2,1)$.
\end{abstract}

1. Introduction. In a bounded domain $\Omega \subset \mathbb{R}^{3}$ with boundary $S$ we consider the Stokes equations with the following initial and boundary conditions

$$
\begin{array}{ll}
\partial_{t} v-\nu \Delta v+\nabla q=f(x, t) & \text { in } \Omega^{T}, \\
\operatorname{div} v=G(x, t) & \text { in } \Omega^{T}, \\
\bar{n} \cdot D(v) \cdot \bar{\tau}_{i}+\gamma v \cdot \bar{\tau}_{i}=b_{i}(i=1,2) & \text { on } S^{T}, \\
v \cdot \bar{n}=b_{3} & \text { on } S^{T}, \\
\left.v\right|_{t=0}=v_{0} & \text { in } \Omega,
\end{array}
$$

where $D(v)=\left\{\nu\left(\partial_{i} v_{j}+\partial_{j} v_{i}\right)\right\}_{\substack{i=1,2,3 \\ j=1,2,3}}$ is the deformation tensor, $v(x, t)(i=1,2,3)$ is the velocity vector, $q(x, t)$ the pressure, $\nu$ the constant viscosity coefficient, $\gamma$ is a positive constant (slip coefficient), $\bar{n}$ the external normal vector to the boundary $S, \bar{\tau}_{i}(i=1,2)$ are the tangent vectors to boundary $S$. The aim of this paper is to prove the existence of regular solutions of (1.1).

The main results of this paper are the following theorems.

2000 Mathematics Subject Classification: 35E99, 76D07.

The paper is in final form and no version of it will be published elsewhere. 
Theorem 1. Let $p \geq 2, S \in W_{p}^{2 s+3}, f \in B_{p, q}^{2 s, s}\left(\Omega^{T}\right), G \in B_{p, q}^{2 s+1, s}\left(\Omega^{T}\right), v_{0} \in B_{p, q}^{2 s+2-2 / p}(\Omega)$, $b_{i} \in B_{p, q}^{2 s+1-1 / p, s+1 / 2 p}\left(S^{T}\right)(i=1,2), b_{3} \in B_{p, q}^{2 s+2-1 / p, s+1-1 / 2 p}\left(S^{T}\right)$ for $s \in \mathbb{R}_{+} \cup\{0\}$. Assume that there exist functions $A, B \in B_{p, q}^{2 s, s}\left(\Omega^{T}\right)$, diamsupp $A<\lambda$, such that the following compatibility conditions hold

$$
D_{x}^{\alpha} \partial_{t}^{\beta+1} G-\operatorname{div}\left(D_{x}^{\alpha} \partial_{t}^{\beta} f\right)=\operatorname{div}\left(D_{x}^{\alpha} \partial_{t}^{\beta} B\right)+D_{x}^{\alpha} \partial_{t}^{\beta} A
$$

for all $\alpha, \beta \geq 0$ such that $|\alpha|+2 \beta \leq[2 s]$. Then there exists a unique solution to the problem (1.1) such that $v \in B_{p, q}^{2 s+2, s+1}\left(\Omega^{T}\right), \nabla q \in B_{p, q}^{2 s, s}\left(\Omega^{T}\right)$ and the following estimate holds

$$
\begin{aligned}
& \|v\|_{B_{p, q}^{2 s+2, s+1}\left(\Omega^{T}\right)}+\|\nabla q\|_{B_{p, q}^{2 s, s}\left(\Omega^{T}\right)} \leq c\left(\|f\|_{B_{p, q}^{2 s, s}\left(\Omega^{T}\right)}+\|B\|_{B_{p, q}^{2 s, s}\left(\Omega^{T}\right)}\right. \\
& \quad+\|A\|_{B_{p, q}^{2 s, s}\left(\Omega^{T}\right)}+\|G\|_{B_{p, q}^{2 s+1, s}\left(\Omega^{T}\right)}+\left\|b_{i}\right\|_{B_{p, q}^{2 s+1-1 / p, s+1 / 2 p}\left(S^{T}\right)} \\
& \left.\quad+\left\|b_{3}\right\|_{B_{p, q}^{2 s+2-1 / p, s+1-1 / 2 p}\left(S^{T}\right)}+\left\|v_{0}\right\|_{B_{p, q}^{2 s+2-2 / p}(\Omega)}+\sum_{i=0}^{[s-1]}\left\|\left.\partial_{t}^{i} f\right|_{t=0}\right\|_{B_{p, q}^{2 s-2 / p-2 i}(\Omega)}\right) .
\end{aligned}
$$

Here, $B_{p, q}^{2 m, m}$ spaces can be replaced by $W_{p}^{2 m, m}$ spaces.

The above theorem presents the existence of regular solutions in the Besov spaces with the Schauder type estimates (the optimal regularity). To prove the result we need the existence in the standard Sobolev spaces $W_{p}^{2 m, m}\left(\Omega^{T}\right)$ with $m \in \mathbb{N}$. Next the interpolation theory applied for model problems in the half space will yield the result in the Besov classes. Another approach to this subject, i.e. interpolation between the regular and weak solution to the Stokes system, implies the next theorem.

THEOREM 2. Let $p>2$, ps $>1$ for $s \in(0,1) \sigma \in(0,1\rangle, p_{*}$ is calculated from (6.58). Assume that $S=\partial \Omega \in W_{p}^{3-1 / p}, f \in L_{p}\left(\Omega^{T}\right), G \in W_{p}^{1,0}\left(\Omega^{T}\right), v_{0} \in W_{p}^{2 s-2 / p}(\Omega), b_{i} \in$ $W_{p}^{2 s-1-1 / p, s-1 / 2-1 / 2 p}\left(S^{T}\right)(i=1,2), b_{3} \in W_{p}^{2 s-1 / p, s-1 / 2 p}\left(S^{T}\right)$. Assume that there exist functions $A, B \in L_{p}\left(\Omega^{T}\right)$, such that the following compatibility condition holds

$$
G_{, t}-\operatorname{div} f=\operatorname{div} B+A,
$$

Then there exists a unique solution to problem (1.1) such that $v \in W_{p}^{2 s, s}\left(\Omega^{T}\right), q \in$ $N_{p_{*}}^{\sigma, 0}\left(\Omega^{T}\right)$ see (Notation, and (6.58)) and the following estimate holds

$$
\begin{aligned}
& \|v\|_{W_{p}^{2 s, s}\left(\Omega^{T}\right)}+\|q\|_{N_{*}^{\sigma, 0}\left(\Omega^{T}\right)} \leq c\left(\|f\|_{L_{p}\left(\Omega^{T}\right)}+\lambda\|A\|_{L_{p}\left(\Omega^{T}\right)}\right. \\
& +\|B\|_{L_{p}\left(\Omega^{T}\right)}+\|G\|_{W_{p}^{1,0}\left(\Omega^{T}\right)}+\left\|b_{i}\right\|_{W_{p}^{2 s-1-1 / p, s-1 / 2-1 / 2 p}\left(S^{T}\right)} \\
& \left.+\left\|b_{3}\right\|_{W_{p}^{2 s-1 / p, s-1 / 2 p}\left(S^{T}\right)}+\left\|v_{0}\right\|_{W_{p}^{2 s-2 / p}(\Omega)}\right) .
\end{aligned}
$$

where spaces $W_{p}^{2 m, m}$ can be replaced by Besov spaces $B_{p, q}^{2 m, m}$.

2. Notation. In our consideration we use the following Sobolev-Slobodetskii and Besov spaces. Sobolev-Slobodetskii spaces $W_{p}^{k, l}\left(Q^{T}\right)$ where $k, l \in R_{+}, p \geq 1, Q^{T}=Q \times(0, T)$ where $Q$ is either $\Omega$ or $S$, have the norm

$$
\|v\|_{W_{p}^{k, l}\left(Q^{T}\right)}=\left(\|v\|_{W_{p}^{k, 0}\left(Q^{T}\right)}^{p}+\|v\|_{W_{p}^{0, l}\left(Q^{T}\right)}^{p}\right)^{1 / p},
$$

where

$$
\|v\|_{W_{p}^{k, 0}\left(Q^{T}\right)}^{p}=\int_{0}^{T}\|v\|_{W_{p}^{k}(Q)}^{p} d t, \quad\|v\|_{W_{p}^{0, l}\left(Q^{T}\right)}^{p}=\int_{Q}\|v\|_{W_{p}^{l}(0, T)}^{p} d x
$$


and

$$
\begin{aligned}
\|v\|_{W_{p}^{k}(Q)}^{p} & =\sum_{|\alpha| \leq[k]}\left\|D_{x}^{\alpha} v\right\|_{L_{p}(Q)}^{p}+\sum_{|\alpha|=[k]} \int_{Q} \int_{Q} \frac{\left|D_{x}^{\alpha} v(x, t)-D_{y}^{\alpha} v(y, t)\right|^{p}}{|x-y|^{s+p \sigma_{1}}} d x d y, \\
\|v\|_{W_{p}^{l}((0, T))}^{p} & =\sum_{i \leq[l]}\left\|\partial_{t}^{i} v\right\|_{L_{p}((0, T))}^{p}+\sum_{|i|=[l]} \int_{0}^{T} \int_{0}^{T} \frac{\left|\partial_{t}^{i} v(x, t)-\partial_{t^{\prime}}^{i} v\left(x, t^{\prime}\right)\right|^{p}}{\left|t-t^{\prime}\right|^{1+p \sigma_{2}}} d t d t^{\prime},
\end{aligned}
$$

where $s \equiv \operatorname{dim} Q, \sigma_{1}=k-[k], \sigma_{2}=l-[l],[m]$ the integral part of $m, D_{x}^{\alpha} \equiv \partial_{x_{1}}^{\alpha_{1}} \ldots \partial_{x_{s}}^{\alpha_{s}}$, where $\alpha=\left(\alpha_{1}, \ldots, \alpha_{s}\right)$ is a multiindex. We will use the Fourier-Laplace transform $F_{t, x}[\phi]\left(\xi, \xi_{0}\right) \equiv \hat{\phi}\left(\xi, \xi_{0}\right)$ where

$$
\hat{\phi}\left(\xi, \xi_{0}\right)=\frac{1}{(2 \pi)^{s / 2}} \int_{0}^{\infty} e^{-s t} \int_{\mathbb{R}^{s}} e^{-i \xi \cdot x} \phi(x, t) d x d t
$$

and the inverse transformation equals

$$
F_{t, x}^{-1}[\hat{\phi}](x, t)=\frac{1}{(2 \pi)^{s / 2}} \int_{0}^{\infty} e^{s t} \int_{\mathbb{R}^{s}} e^{i \xi \cdot x} \hat{\phi}\left(\xi, \xi_{0}\right) d \xi d \xi_{0},
$$

where $s=i \xi_{0}, \xi=\left(\xi_{1}, \ldots, \xi_{s}\right), x=\left(x_{1}, \ldots, x_{s}\right)$ and $x \cdot \xi=\sum_{j=1}^{s} x_{j} \xi_{j}$.

Let us introduce the following spaces, $\stackrel{\circ}{{ }^{2}} \underset{p}{2 m, m}\left(Q^{T}\right)$ and $\stackrel{\circ}{B}_{p, q}^{2 m, m}\left(Q^{T}\right)$ where

$$
\begin{aligned}
& \stackrel{\circ}{W}_{p}^{2 m, m}\left(Q^{T}\right)=\left\{v: v \in W_{p}^{2 m, m}\left(Q^{T}\right),\left.v\right|_{t=0}=0\right\}, \\
& \stackrel{\circ}{B}_{p, q}^{2 m, m}\left(Q^{T}\right)=\left\{v: v \in B_{p, q}^{2 m, m}\left(Q^{T}\right),\left.v\right|_{t=0}=0\right\} .
\end{aligned}
$$

We introduce also $N_{p}^{\sigma, 0}\left(Q^{t}\right)$ and Besov spaces $B_{p, q}^{m, n}\left(Q^{T}\right)$, where

$$
N_{p_{*}}^{\sigma, 0}\left(Q^{T}\right)=\left\{f \in M_{p_{*}}^{\sigma, 0}\left(Q^{T}\right): \int_{Q} f d x=0\right\},
$$

for

$$
M_{p_{*}}^{\sigma, 0}\left(Q^{T}\right)=\left\{f:\left|\int_{0}^{T} \int_{Q} \int_{Q} \frac{\left|f(x, t)-f\left(x^{\prime}, t\right)\right|^{p_{*}}}{\left|x-x^{\prime}\right|^{N+\sigma p_{*}}} d x d x^{\prime} d t\right|<\infty\right\}
$$

for $\sigma \in[0,1]$. Now we introduce Besov spaces as

$$
B_{p, q}^{m, n}\left(Q^{T}\right)=\left\{v: Q^{T} \rightarrow \mathbb{R}^{s},\|v\|_{B_{p, q}^{m, n}\left(Q^{T}\right)}<\infty\right\}
$$

for

$$
\begin{aligned}
\|v\|_{B_{p, q}^{m, n}\left(Q^{T}\right)}= & \|v\|_{L_{p}\left(Q^{T}\right)}+\sum_{|\alpha| \leq k}\left\||h|^{-(m-k)} \Delta_{h}^{l} D^{\alpha} v\right\|_{L_{q}^{*}\left(Q \delta, L_{p}(0, T)\right)} \\
& +\sum_{|\beta| \leq t}\left\||h|^{-(n-t)} \Delta_{h}^{l} \partial_{t}^{\beta} v\right\|_{L_{q}^{*}\left((0, \delta), L_{p}(Q)\right)}
\end{aligned}
$$

for $\Delta_{h}^{l} g=\Delta_{h}^{l-1} \Delta_{h} g=\Delta_{h}^{l-1}(g(x+h)-g(x))$ and

$$
L_{q}^{*}(A) \equiv\left\{\phi: \mid\left(\int_{0}^{\infty}\|\phi(\tau)\|_{A}^{q} \frac{d t}{t}\right)^{1 / q}<\infty\right\}
$$

where

$$
\|\phi\|_{L_{q}^{*}\left(Q \delta, L_{p}\left(Q^{T}\right)\right)} \equiv\left(\int_{Q \delta}\|\phi\|_{L_{p}\left(Q^{T}\right)}^{q} \frac{d x d t}{|t||x|^{s}}\right)^{1 / q}
$$


for

$$
Q \delta \equiv\left\{(x, t): x=\left(x_{1}, x_{2}, \ldots, x_{s}\right): 0<x_{i}<\delta, 0<t<\delta\right\} .
$$

In the proofs we will use the following results

Theorem 2.1 (see [1]). Let $p>3, S \in W_{p}^{2-1 / p}, f \in L_{p}\left(\Omega^{T}\right) v_{0} \in W_{p}^{2-2 / p}(\Omega), b_{i} \in$ $W_{p}^{1-1 / p, 1 / 2-1 / 2 p}\left(S^{T}\right), \quad(i=1,2), b_{3} \in W_{p}^{2-1 / p, 1-1 / 2 p}\left(S^{T}\right), G \in W_{p}^{1,0}\left(\Omega^{T}\right)$. Suppose that there exist functions $A, B$ such that $A, B \in L_{p}\left(\Omega^{T}\right)$, $\operatorname{diam} \operatorname{supp} A<\lambda$, and $G_{, t}-\operatorname{div} f=$ $A+\operatorname{div} B$. Then there exists a unique solution of system (1.1) such that $v \in W_{p}^{2,1}\left(\Omega^{T}\right)$, $\nabla q \in L_{p}\left(\Omega^{T}\right)$ and the following estimate holds

$$
\begin{aligned}
\|v\|_{W_{p}^{2,1}\left(\Omega^{T}\right)}+ & \|\nabla q\|_{L p\left(\Omega^{T}\right)} \leq c\left(\|f\|_{L p\left(\Omega^{T}\right)}+\|B\|_{L p\left(\Omega^{T}\right)}+\lambda\|A\|_{L p\left(\Omega^{T}\right)}+\|G\|_{W_{p}^{1,0}\left(\Omega^{T}\right)}\right. \\
& \left.+\left\|b_{i}\right\|_{W_{p}^{1-1 / p, 1 / 2-1 / 2 p}\left(S^{T}\right)}+\left\|b_{3}\right\|_{W_{p}^{2-1 / p, 1-1 / 2 p}\left(S^{T}\right)}+\left\|v_{0}\right\|_{W_{p}^{2-2 / p}(\Omega)}\right) .
\end{aligned}
$$

Lemma 2.2 (see [11]). Let $\left(A_{0},\|\cdot\|_{A_{0}}\right),\left(A_{1}\|\cdot\|_{A_{1}}\right)$ be Banach spaces. Let us consider the following functional $K\left(s, a, A_{0} A_{1}\right)=\inf _{a=a_{0}+a_{1}}\left(\left\|a_{0}\right\|_{A_{0}}+s\left\|a_{1}\right\|_{A_{1}}\right)$, for $s \in(0, \infty)$. Let $1 \leq q \leq \infty$ and $\theta \in(0,1)$. We define the following space

$$
\left(A_{0}, A_{1}\right)_{\theta, q}=\left\{a \mid a \in A_{0}+A_{1}:\|a\|_{\left(A_{0}, A_{1}\right)_{\theta, q}}<\infty\right\},
$$

where $A_{0}+A_{1} \equiv\left\{a \mid a \in \mathcal{H}\right.$ and $\left.a=a_{0}+a_{1}: a_{i} \in A_{i}\right\} A_{0}, A_{1} \subset \mathcal{H}$ is a Hausdorff linear space,

$$
\|a\|_{\left(A_{0}, A_{1}\right)_{\theta, q}}=\left(\int_{0}^{\infty}\left(s^{-\theta} K\left(s, a, A_{0}, A_{1}\right)\right)^{q} \frac{d s}{s}\right)^{1 / q}
$$

for $1 \leq q<\infty$ and

$$
\|a\|_{\left(A_{0}, A_{1}\right)_{\theta, \infty}}=\sup _{0<s<\infty} s^{-\theta} K\left(s, a, A_{0}, A_{1}\right)
$$

for $q=\infty$. Then $K\left(s, a, A_{0}, A_{1}\right)$ is a norm in $A_{0}+A_{1}$ and $\left(A_{0}, A_{1}\right)_{\theta, q}$ is a Banach space such that the following topological embeddings hold

$$
A_{0} \cap A_{1} \subset\left(A_{0}, A_{1}\right)_{\theta, q} \subset A_{0}+A_{1} .
$$

Proposition 2.3 (see $[4,8,9]$ ). Let $1 \leq p_{i} \leq \infty, 0<\theta<1$ and $k_{i} \in \mathbb{Z}_{+} \cup\{0\} i=0,1$. Then the following interpolation holds

$$
\left(W_{p_{0}}^{2 k_{0}, k_{0}}\left(\mathbb{R}_{+}^{N+1}\right), W_{p_{1}}^{2 k_{1}, k_{1}}\left(\mathbb{R}_{+}^{N+1}\right)\right)_{\theta, p}=W_{p}^{2 s, s}\left(\mathbb{R}_{+}^{N+1}\right)
$$

$k_{0} \neq k_{1}$, for $s=(1-\theta) k_{0}+\theta k_{1}, \frac{1}{p}=\frac{1-\theta}{p_{0}}+\frac{\theta}{p_{1}}$, and

$$
\left(W_{p_{i}}^{2 k_{0}, k_{0}}\left(\mathbb{R}_{+}^{N+1}\right), W_{p_{i}}^{2 k_{1}, k_{1}}\left(\mathbb{R}_{+}^{N+1}\right)\right)_{\theta, q}=B_{p_{i}, q}^{2 s, s}\left(\mathbb{R}_{+}^{N+1}\right)
$$

for $k_{0} \neq k_{1}$ and $s=(1-\theta) k_{0}+\theta k_{1} ; \mathbb{R}_{+}^{m}=\mathbb{R}^{m-1} \times(0, \infty)$.

Theorem 2.4 (see $[3,5,6,7,11]$ ). Let $A_{0}, A_{1}$ be an interpolation couple of Banach spaces. Let $T$ be a linear continuous operator $T: A_{0}+A_{1} \rightarrow B_{0}+B_{1}$ such that $T: A_{j} \rightarrow B_{j}$ as a restriction is also continuous and linear. Assume that $\left(A_{0}, A_{1}\right)_{\theta, p}$ and $\left(B_{0}, B_{1}\right)_{\theta, p}$ are well defined interpolation spaces. Then for $v \in\left(A_{0}, A_{1}\right)_{\theta, p}$ there exists $T v \in\left(B_{0}, B_{1}\right)_{\theta, p}$, $T:\left(A_{0}, A_{1}\right)_{\theta, p} \rightarrow\left(B_{0}, B_{1}\right)_{\theta, p}$ is a linear continuous and the following estimate holds

$$
\|T v\|_{\left(B_{0}, B_{1}\right)_{\theta, p}} \leq c\|v\|_{\left(A_{0}, A_{1}\right)_{\theta, p}},
$$

where constant $c$ is equal $\|T\|_{A_{0} \rightarrow B_{0}}^{1-\theta}\|T\|_{A_{1} \rightarrow B_{1}}^{\theta}$. 
LEMma 2.5 (see [12]). Assume that $v_{0} \in W_{p}^{2 k+2-2 / p}\left(\mathbb{R}^{3}\right)$ for $k \in \mathbb{N}$. Then exists an extension $\phi$ of $v_{0}$ such that $\phi \in W_{p}^{2 k+2, k+1}\left(\mathbb{R}^{4}\right),\left.\phi\right|_{t=0}=v_{0}$ and the following inequality holds

$$
\|\phi\|_{W_{p}^{2 k+2, k+1}\left(\mathbb{R}^{4}\right)} \leq c\left\|v_{0}\right\|_{W_{p}^{2 k+2-2 / p}\left(\mathbb{R}^{3}\right)} .
$$

3. Problem in half space. The first step to solve the problem (1.1) in a Besov space is to consider this system in the half space $x_{3} \geq 0$,

$$
\begin{aligned}
& v_{, t}-\nu \Delta v+\nabla q=f(x, t), \\
& \operatorname{div} v=G(x, t), \\
& v_{1, x_{3}}+\left.v_{3, x_{1}}\right|_{x_{3}=0}=b_{1}\left(x^{\prime}, 0, t\right), \\
& v_{2, x_{3}}+\left.v_{3, x_{2}}\right|_{x_{3}=0}=b_{2}\left(x^{\prime}, 0, t\right), \\
& \left.v_{3}\right|_{x_{3}=0}=b_{3}\left(x^{\prime}, 0, t\right), \\
& \left.v\right|_{t=0}=v_{0}(x),
\end{aligned}
$$

for $x=\left(x_{1}, x_{2}, x_{3}\right) ; x^{\prime}=\left(x_{1}, x_{2}\right)$.

Lemma 3.1. Let $f \in W_{p}^{2 k, k}\left(\mathcal{D}_{T}^{4}\right), G \in W_{p}^{2 k+1, k}\left(\mathcal{D}_{T}^{4}\right), v_{0} \in W_{p}^{2 k+2-2 / p}\left(\mathbb{R}_{+}^{3}\right), b_{i} \in$ $W_{p}^{2 k+1-1 / p, k+1 / 2-1 / 2 p}\left(\mathbb{R}_{+}^{3}\right)(i=1,2), b_{3} \in W_{p}^{2 k+2-1 / p, k+1-1 / 2 p}\left(\mathbb{R}_{+}^{3}\right)$, for $k \in Z_{+}$. Assume that there exist functions $A, B \in W_{p}^{2 k, k}\left(\mathcal{D}_{+}^{4}\right)$ such that the following compatibility conditions $D_{x}^{\alpha} \partial_{t}^{\beta+1} G-\operatorname{div} D_{x}^{\alpha} \partial_{t}^{\beta} f=\operatorname{div} D_{x}^{\alpha} \partial_{t}^{\beta} B+D_{x}^{\alpha} \partial_{t}^{\beta} A$, where $|\alpha|+2 \beta \leq 2 k$ hold. Then there exists a unique solution of problem $(3.1)$ such that $v \in W_{p}^{2 k+2, k+1}\left(\mathcal{D}_{T}^{4}\right)$, $\nabla q \in W_{p}^{2 k, k}\left(\mathcal{D}_{T}^{4}\right)$ for $\mathcal{D}_{T}^{4}=\mathbb{R}^{2} \times \mathbb{R}_{+} \times \mathbb{R}_{+} ; \mathbb{R}_{+}=(0, \infty)$ and the following estimate holds

$$
\begin{aligned}
& \|v\|_{W_{p}^{2 k+2, k+1}\left(\mathcal{D}_{T}^{4}\right)}+\|\nabla q\|_{W_{p}^{2 k, k}\left(\mathcal{D}_{T}^{4}\right)} \leq c\left(\|f\|_{W_{p}^{2 k, k}\left(\mathcal{D}_{T}^{4}\right)}+\|B\|_{W_{p}^{2 k, k}\left(\mathcal{D}_{T}^{4}\right)}\right. \\
& \quad+\|A\|_{W_{p}^{2 k, k}\left(\mathcal{D}_{T}^{4}\right)}+\|G\|_{W_{p}^{2 k+1, k}\left(\mathcal{D}_{T}^{4}\right)}+\left\|b_{i}\right\|_{W_{p}^{2 k+1-1 / p, k+1 / 2-1 / 2 p}\left(\mathbb{R}_{+}^{3}\right)} \\
& \left.\quad+\left\|b_{3}\right\|_{W_{p}^{2 k+2-1 / p, k+1-1 / 2 p}\left(\mathbb{R}_{+}^{3}\right)}+\left\|v_{0}\right\|_{W_{p}^{2 k+2-2 / p}\left(\mathbb{R}_{+}^{3}\right)}\right) .
\end{aligned}
$$

Proof. We need first to consider the following problem

$$
\begin{aligned}
& v_{, t}-\nu \Delta v+\nabla q=0, \\
& \operatorname{div} v=0, \\
& v_{1, x_{3}}+\left.v_{3, x_{1}}\right|_{x_{3}=0}=b_{1}, \\
& v_{2, x_{3}}+\left.v_{3, x_{2}}\right|_{x_{3}=0}=b_{2}, \\
& \left.v_{3}\right|_{x_{3}=0}=b_{3}, \\
& \left.v\right|_{t=0}=0,
\end{aligned}
$$

for $b_{i} \in W_{p}^{2 k+1-1 / p, k+1 / 2-1 / 2 p}\left(\mathbb{R}_{+}^{3}\right), b_{3} \in W_{p}^{2 k+2-1 / p, k+1-1 / 2 p}\left(\mathbb{R}_{+}^{3}\right)(i=1,2)$. Next, we consider formally the following system 


$$
\begin{aligned}
& \tilde{v}_{, t}-\nu \Delta \tilde{v}+\nabla \tilde{q}=0, \\
& \operatorname{div} \tilde{v}=0, \\
& \tilde{v}_{i, x_{3}}+\left.\tilde{v}_{3, x_{i}}\right|_{x_{3}=0}=\tilde{b}_{i}, \\
& \left.\tilde{v}_{3}\right|_{x_{3}=0}=\tilde{b}_{3}, \\
& \left.\tilde{v}\right|_{t=0}=0,
\end{aligned}
$$

where $\tilde{v}=\partial_{x^{\prime}}^{\alpha} v, \tilde{q}=\partial_{x^{\prime}}^{\alpha} p, \tilde{b}_{j}=\partial_{x^{\prime}}^{\alpha} b_{j},(j=1,2,3) \partial_{x^{\prime}}^{\alpha}=\frac{\partial^{|\alpha|}}{\partial_{x_{1}}^{\alpha_{1}} \partial_{x_{2}}^{\alpha_{2}}} ;|\alpha|=\alpha_{1}+\alpha_{2}$, for $|\alpha| \leq 2 k$. Now using Theorem 2.1 we arrive at the following

$$
\|\tilde{v}\|_{W_{p}^{2,1}\left(\mathcal{D}_{T}^{4}\right)}+\|\nabla \tilde{q}\|_{L_{p}\left(\mathcal{D}_{T}^{4}\right)} \leq c\left\|b_{i}\right\|_{W_{p}^{1-1 / p, 1 / 2-1 / 2 p}\left(\mathbb{R}_{+}^{3}\right)}+c\left\|b_{3}\right\|_{W_{p}^{2-1 / p, 1-1 / 2 p}\left(\mathbb{R}_{+}^{3}\right)} .
$$

At this point we need to estimate the derivatives with respect to $x_{3}, t$ and the mixed derivatives of the form $D_{x}^{\alpha} \partial_{t}^{i} v, D_{x}^{\alpha} \partial_{t}^{i} q$ for $|\alpha|+2 i \leq 2 k$. In order to do it we write $\tilde{q}$ and $\tilde{v}$ in explicit form. From [1] we have $\tilde{q}=F_{t, x^{\prime}}^{-1}(\hat{\tilde{q}})$, where

$$
\begin{aligned}
& \hat{\tilde{q}}=\sum_{j=1}^{2} \frac{i \xi_{i}}{\left|\xi^{\prime}\right|} \hat{\tilde{b}}_{j} e^{-\left|\xi^{\prime}\right| x_{3}}+\frac{\left(\left|\xi^{\prime}\right|^{2}+r^{2}\right)}{\left|\xi^{\prime}\right|} \hat{\tilde{b}}_{3} e^{-\left|\xi^{\prime}\right| x_{3}}, \\
& \tilde{v}=\frac{1}{(2 \pi)^{3 / 2}} \int_{0}^{\infty} e^{s t} \int_{R^{2}} e^{i x^{\prime} \xi^{\prime}} \hat{\tilde{v}}\left(\xi^{\prime}, s, x_{3}\right) d \xi^{\prime} d \xi_{0},
\end{aligned}
$$

where $\hat{\tilde{v}}\left(\xi^{\prime}, s, x_{3}\right)=\hat{\tilde{v}}_{i} \bar{e}_{i}$ for

$$
\begin{aligned}
\hat{\tilde{v}}_{1}= & {\left[\left(\frac{1}{r}+\frac{\xi_{1}^{2}}{s}\left(\frac{1}{\left|\xi^{\prime}\right|}-\frac{1}{r}\right)\right) e^{-r x_{3}}+\frac{\xi_{1}^{2}}{s} \frac{\left|\xi^{\prime}\right|-r}{\left|\xi^{\prime}\right|}\left(\frac{e^{-\left|\xi^{\prime}\right| x_{3}}-e^{-r x_{3}}}{\left|\xi^{\prime}\right|-r}\right)\right] \hat{\tilde{b}}_{1} } \\
& +\left(\frac{1}{\left|\xi^{\prime}\right|}-\frac{1}{r}\right) \frac{\xi_{1} \xi_{2}}{s} \hat{\tilde{b}}_{2} e^{-r x_{3}}+\frac{\xi_{1} \xi_{2}}{s} \frac{\left(\left|\xi^{\prime}\right|-r\right)}{\left|\xi^{\prime}\right|}\left(\frac{e^{-\left|\xi^{\prime}\right| x_{3}}-e^{-r x_{3}}}{\left|\xi^{\prime}\right|-r}\right) \hat{\tilde{b}}_{2} \\
& +\frac{i \xi_{1}}{r} \hat{\tilde{b}}_{3} e^{-r x_{3}}+\left(\frac{1}{r}-\frac{1}{\left|\xi^{\prime}\right|}\right) \frac{i \xi_{1}\left(\left|\xi^{\prime}\right|^{2}+r^{2}\right)}{s} e^{-r x_{3} \hat{\tilde{b}}_{3}} \\
& -\frac{\left(\left|\xi^{\prime}\right|-r\right)}{\left|\xi^{\prime}\right|} \frac{i \xi_{1}\left(\left|\xi^{\prime}\right|^{2}+r^{2}\right)}{s}\left(\frac{e^{-\left|\xi^{\prime}\right| x_{3}}-e^{-r x_{3}}}{\left|\xi^{\prime}\right|-r}\right) \hat{b}_{3}, \\
\hat{\tilde{v}}_{2}= & \left(\frac{1}{\left|\xi^{\prime}\right|}-\frac{1}{r}\right) \frac{\xi_{1} \xi_{2}}{s} \hat{\tilde{b}}_{1} e^{-r x_{3}}+\frac{\xi_{1} \xi_{2}}{s} \frac{\left|\xi^{\prime}\right|-r}{\left|\xi^{\prime}\right|}\left(\frac{1}{\left|\xi^{\prime}\right|-r}\right)\left(e^{-\left|\xi^{\prime}\right| x_{3}}-e^{-r x_{3}}\right) \hat{\tilde{b}}_{1} \\
& -\frac{1}{r} \hat{\tilde{s}}_{2} e^{-r x_{3}}+\frac{\xi_{2}}{s}\left(\frac{1}{\left|\xi^{\prime}\right|}-\frac{1}{r}\right) e^{-r x_{3}} \hat{\tilde{b}}_{2}+\frac{\xi_{2}^{2}}{s} \frac{\left(\left|\xi^{\prime}\right|-r\right)}{\left|\xi^{\prime}\right|}\left(\frac{e^{-\left|\xi^{\prime}\right| x_{3}}-e^{-r x_{3}}}{\left|\xi^{\prime}\right|-r}\right) \hat{\tilde{b}}_{2} \\
& +\frac{i \xi_{2}}{r} \hat{\tilde{b}}_{3} e^{-r x_{3}}+\left(\frac{1}{\left|\xi^{\prime}\right|^{2}}-\frac{1}{r^{2}}\right) \frac{\left|\xi^{\prime}\right|^{2}\left(\left|\xi^{\prime}\right|^{2}+r^{2}\right)}{s} \hat{\tilde{b}}_{3} e^{-r x_{3}} \\
& +\frac{\left(\left|\xi^{\prime}\right|-r\right)\left(\left|\xi^{\prime}\right|^{2}+r^{2}\right)}{s}\left(\frac{e^{-\left|\xi^{\prime}\right| x_{3}}-e^{-r x_{3}}}{\left|\xi^{\prime}\right|-r}\right) \hat{\tilde{b}}_{3}, \\
\hat{\tilde{v}}_{3}= & \frac{i \xi_{1}}{r^{2}} \hat{\tilde{b}}_{1} e^{-r x_{3}}+\left(\frac{1}{\left|\xi^{\prime}\right|^{2}}-\frac{1}{r^{2}}\right) \frac{i \xi_{1}}{s}\left|\xi^{\prime}\right|^{2} \hat{\tilde{b}}_{1} e^{-r x_{3}}+\frac{i \xi_{1}}{s}\left|\xi^{\prime}\right|^{2} \frac{\left|\xi^{\prime}\right|-r}{\left|\xi^{\prime}\right|^{2}}\left(\frac{e^{-\left|\xi^{\prime}\right| x_{3}}-e^{-r x_{3}}}{\left|\xi^{\prime}\right|-r}\right) \hat{\tilde{b}}_{1}
\end{aligned}
$$




$$
\begin{aligned}
& +\frac{i \xi_{2}}{r^{2}} \hat{\tilde{b}}_{2} e^{-r x_{3}}+\left(\frac{1}{\left|\xi^{\prime}\right|}-\frac{1}{r^{2}}\right) \frac{i \xi_{2}}{s}\left|\xi^{\prime}\right| \hat{\tilde{b}}_{2} e^{-r x_{3}}+\frac{i \xi_{2}\left|\xi^{\prime}\right|}{s} \frac{\left|\xi^{\prime}\right|-r}{\left|\xi^{\prime}\right|}\left(\frac{e^{-\left|\xi^{\prime}\right| x_{3}}-e^{-r x_{3}}}{\left|\xi^{\prime}\right|-r}\right) \hat{\tilde{b}}_{2} \\
& -\frac{\left|\xi^{\prime}\right|^{2}}{r^{2}} \hat{\tilde{b}}_{3} e^{-r x_{3}}+\left(\frac{1}{\left|\xi^{\prime}\right|^{2}}-\frac{1}{r^{2}}\right) \frac{\left|\xi^{\prime}\right|^{2}\left(\left|\xi^{\prime}\right|^{2}+r^{2}\right)}{s} \hat{\tilde{b}}_{3} e^{-r x_{3}} \\
& +\frac{\left(\left|\xi^{\prime}\right|-r\right)\left(\left|\xi^{\prime}\right|^{2}+r^{2}\right)}{s}\left(\frac{e^{-\left|\xi^{\prime}\right| x_{3}}-e^{-r x_{3}}}{\left|\xi^{\prime}\right|-r}\right) \hat{\tilde{b}}_{3},
\end{aligned}
$$

where $r^{2} \equiv\left|\xi^{\prime}\right|^{2}+s$, and $r \in\left(-\frac{\pi}{4}, \frac{\pi}{4}\right), s=i \xi_{0}$. First we consider $\partial_{t}^{j} \nabla \tilde{q}$, for $|\alpha|+2 j \leq 2 k$; $|\alpha|=\alpha_{1}+\alpha_{2}$. This implies

$$
\begin{aligned}
\partial_{t}^{i} \nabla \tilde{q}= & \frac{1}{(2 \pi)^{3 / 2}} \nabla \partial_{t}^{i} \int_{0}^{\infty} e^{s t} \int_{R^{2}} e^{i x^{\prime} \xi^{\prime}} \hat{\tilde{p}}\left(\xi^{\prime}, s, x_{3}\right) d \xi_{0} d \xi^{\prime} \\
= & \nabla \partial_{t}^{j} F_{t, x^{\prime}}^{-1}\left(\sum_{j=1}^{2} \frac{i \xi_{j}}{\left|\xi^{\prime}\right|} \hat{\tilde{b}}_{j} e^{-\left|\xi^{\prime}\right| x_{3}}\right)+\nabla \partial_{t}^{i} F_{x, t}^{-1}\left(\frac{\left(\left|\xi^{\prime}\right|^{2}+r^{2}\right)}{\left|\xi^{\prime}\right|} \hat{\tilde{b}}_{3} e^{-\left|\xi^{\prime}\right| x_{3}}\right) \\
= & \nabla\left[F_{t, x^{\prime}}^{-1}\left(\frac{\left(\left|\xi^{\prime}\right|^{2}+r^{2}\right)}{\left|\xi^{\prime}\right|}\left(i \xi_{0}\right)^{j} \hat{\tilde{b}}_{3} e^{-\left|\xi^{\prime}\right| x_{3}}\right)+\sum_{m=1}^{2} F_{t, x}^{-1}\left(\frac{i \xi_{m}}{\left|\xi^{\prime}\right|} \hat{\tilde{b}}_{m}\left(i \xi_{0}\right)^{j} e^{-\left|\xi^{\prime}\right| x_{3}}\right)\right] \\
= & \nabla\left[f_{t, x^{\prime}}^{-1}\left(i \xi_{0}\right)^{j} \tilde{\tilde{b}}_{3} * F_{t, x^{\prime}}^{-1}\left(\frac{\left(\left|\xi^{\prime}\right|^{2}+r^{2}\right)}{\left|\xi^{\prime}\right|} e^{-\left|\xi^{\prime}\right| x_{3}}\right)\right. \\
& \left.+\sum_{m=1}^{2} F_{t, x^{\prime}}^{-1}\left(\left(i \xi_{0}\right)^{j} \hat{\tilde{b}}_{m}\right) * F_{t, x^{\prime}}^{-1}\left(\frac{i \xi_{m}}{\left|\xi^{\prime}\right|} e^{-\left|\xi^{\prime}\right| x_{3}}\right)\right] \\
= & \nabla\left[\partial_{t}^{j} \hat{\tilde{b}}_{3} * F_{t, x^{\prime}}^{-1}\left(\frac{\left(\left|\xi^{\prime}\right|^{2}+r^{2}\right)}{\left|\xi^{\prime}\right|} e^{-\left|\xi^{\prime}\right| x_{3}}\right)\right]+\nabla\left[\sum_{m=1}^{2} \partial_{t}^{j} \hat{\tilde{b}}_{m} * F_{t, x^{\prime}}^{-1}\left(\frac{i \xi_{m}}{\left|\xi^{\prime}\right|} e^{-\left|\xi^{\prime}\right| x_{3}}\right)\right],
\end{aligned}
$$

where $*$ denotes convolution.

Inserting $g_{k} \equiv \partial_{t}^{j} \tilde{b}_{k}(k=1,2,3)$ one can see from [1] that

$$
\begin{aligned}
& \left\|\nabla \partial_{t}^{j} \tilde{q}\right\|_{L_{p}\left(\mathcal{D}_{T}^{4}\right)} \leq c\left(\left\|g_{i}\right\|_{W_{p}^{1-1 / p, 1 / 2-1 / 2 p}\left(\mathbb{R}_{+}^{3}\right)}+\left\|g_{3}\right\|_{W_{p}^{2-1 / p, 1-1 / 2 p}\left(\mathbb{R}_{+}^{3}\right)}\right) \\
& \leq c\left(\left\|\partial_{t}^{j} \tilde{b}_{i}\right\|_{W_{p}^{1-1 / p, 1 / 2-1 / 2 p}\left(\mathbb{R}_{+}^{3}\right)}+\left\|\partial_{t}^{j} \tilde{b}_{3}\right\|_{W_{p}^{2-1 / p, 1-1 / 2 p}\left(\mathbb{R}_{+}^{3}\right)}\right) \\
& \leq c\left(\left\|b_{i}\right\|_{W_{p}^{2 k+1-1 / p, k+1 / 2-1 / 2 p}\left(\mathbb{R}_{+}^{3}\right)}+\left\|b_{3}\right\|_{W_{p}^{2 k+2-1 / p, k+1-1 / 2 p}\left(\mathbb{R}_{+}^{3}\right)}\right) .
\end{aligned}
$$

In this same way we find the estimates of $\partial_{t}^{j} \tilde{v}$. The next step is to find the estimate of $\partial_{x_{3}}^{\alpha_{3}} \nabla \tilde{q}$ for $\alpha_{1}+\alpha_{2}+\alpha_{3} \leq 2 k$. First, we calculate $\partial_{x_{3}}^{\alpha_{3}} \tilde{q}$, where

$$
\begin{aligned}
\partial_{x_{3}}^{\alpha_{3}} \tilde{q}= & \sum_{m=1}^{2} \partial_{x_{3}}^{\alpha_{3}} F_{t, x^{\prime}}^{-1}\left(\frac{i \xi_{m}}{\left|\xi^{\prime}\right|} \hat{\tilde{b}}_{m} e^{-\left|\xi^{\prime}\right| x_{3}}\right)+\partial_{x_{3}}^{\alpha_{3}} F_{t, x^{\prime}}^{-1}\left(\frac{\left|\xi^{\prime}\right|^{2}+r^{2}}{\left|\xi^{\prime}\right|} \hat{\tilde{b}}_{3} e^{-\left|\xi^{\prime}\right| x_{3}}\right) \\
= & \sum_{m=1}^{2} F_{t, x^{\prime}}^{-1}\left(\frac{i \xi_{m}}{\left|\xi^{\prime}\right|} \hat{\tilde{b}}_{m}(-1)^{\alpha_{3}}\left|\xi^{\prime}\right|^{\alpha_{3}} e^{-\left|\xi^{\prime}\right| x_{3}}\right)+F_{t, x^{\prime}}^{-1}\left(\frac{\left|\xi^{\prime}\right|^{2}+r^{2}}{\left|\xi^{\prime}\right|} \hat{\tilde{b}}_{3}(-1)^{\alpha_{3}}\left|\xi^{\prime}\right|^{\alpha_{3}} e^{-\left|\xi^{\prime}\right| x_{3}}\right) \\
& \sim \frac{1}{(2 \pi)^{3 / 2}} \int_{0}^{\infty} e^{s t} \int_{R^{2}} e^{i x^{\prime} \cdot \xi^{\prime}}\left|\xi^{\prime}\right|^{\alpha_{3}+1} \hat{\tilde{b}}_{3} e^{-\left|\xi^{\prime}\right| x_{3}} d \xi_{0} d \xi^{\prime} \\
& +\frac{1}{(2 \pi)^{3 / 2}} \sum_{m=1}^{2} \int_{0}^{\infty} e^{s t} \int_{R^{2}} e^{i x^{\prime} \cdot \xi^{\prime}}\left|\xi^{\prime}\right|^{\alpha_{3}} \hat{\tilde{b}}_{m} e^{-\left|\xi^{\prime}\right| x_{3}} d \xi_{0} d \xi^{\prime},
\end{aligned}
$$

where $\sim$ means proportional to the constant. 
Now, we need to estimate $\nabla \partial_{x_{3}}^{\alpha_{3}} \tilde{q}$ in the $L_{p}$-norm. First, we calculate $\nabla \partial_{x_{3}}^{\alpha} \tilde{q}$, where

$$
\begin{aligned}
\nabla \partial_{x_{3}}^{\alpha_{3}} \tilde{q} \sim & \frac{1}{(2 \pi)^{3 / 2}} \int_{0}^{\infty} e^{s t} \int_{R^{2}} e^{i x^{\prime} \cdot \xi^{\prime}}\left|\xi^{\prime}\right|^{\alpha_{3}+2} \hat{\tilde{b}}_{3} e^{-\left|\xi^{\prime}\right| x_{3}} d \xi_{0} d \xi^{\prime} \\
& +\frac{1}{(2 \pi)^{3 / 2}} \sum_{m=1}^{2} \int_{0}^{\infty} e^{s t} \int_{R^{2}} e^{i x^{\prime} \cdot \xi^{\prime}} \hat{\tilde{b}}_{m} e^{-\left|\xi^{\prime}\right| x_{3}} d \xi_{0} d \xi^{\prime} .
\end{aligned}
$$

Inserting $\hat{b}_{m}^{*} \equiv\left|\xi^{\prime}\right|^{\alpha_{3}} \hat{\tilde{b}}_{m}(m=1,2,3)$, we have the following quantity

$$
\begin{aligned}
\nabla \partial_{x_{3}}^{\alpha_{3}} \tilde{q} \sim & \frac{1}{(2 \pi)^{3 / 2}} \int_{0}^{\infty} e^{s t} \int_{R^{2}} e^{i x^{\prime} \cdot \xi^{\prime}}\left|\xi^{\prime}\right|^{2} \hat{b}_{3}^{*} e^{-\left|\xi^{\prime}\right| x_{3}} d \xi_{0} d \xi^{\prime} \\
& +\frac{1}{(2 \pi)^{3 / 2}} \int_{0}^{\infty} e^{s t} \int_{R^{2}} e^{i x^{\prime} \cdot \xi^{\prime}}\left|\xi^{\prime}\right| \hat{b}_{m}^{*} e^{-\left|\xi^{\prime}\right| x_{3}} d \xi_{0} d \xi^{\prime}, \quad m=1,2
\end{aligned}
$$

and now using [1] we obtain

$$
\begin{aligned}
& \left\|\nabla \partial_{x_{3}}^{\alpha_{3}} \tilde{q}\right\|_{L_{p}\left(\mathcal{D}_{T}^{4}\right)} \leq c\left(\left\|b_{m}^{*}\right\|_{W_{p}^{1-1 / p, 1 / 2-1 / 2 p}\left(\mathbb{R}_{+}^{3}\right)}+\left\|b_{3}^{*}\right\|_{W_{p}^{2-1 / p, 1-1 / 2 p}\left(\mathbb{R}_{+}^{3}\right)}\right) \\
& =c\left(\left\|D_{x^{\prime}}^{\alpha_{3}} \tilde{b}_{m}\right\|_{W_{p}^{1-1 / p, 1 / 2-1 / 2 p}\left(\mathbb{R}_{+}^{3}\right)}+\left\|D_{x^{\prime}}^{\alpha_{3}} \tilde{b}_{3}\right\|_{W_{p}^{2-1 / p, 1-1 / 2 p}\left(\mathbb{R}_{+}^{3}\right)}\right)
\end{aligned}
$$

where $\tilde{b}_{m}=D_{x^{\prime}}^{\alpha^{\prime}} b_{m}, \tilde{b}_{3}=D_{x^{\prime}}^{\alpha^{\prime}} b_{3}, x^{\prime}=\left(x_{1}, x_{2}\right) \alpha^{\prime}=\left(\alpha_{2}, \alpha_{2}\right) ; D_{x^{\prime}}^{\alpha^{\prime}}=\frac{\partial^{\left|\alpha^{\prime}\right|}}{\partial_{x_{1}}^{\alpha_{1}} \partial_{x_{2}}^{\alpha_{2}}} ;\left|\alpha^{\prime}\right|=\alpha_{1}+\alpha_{2}$.

Due to the previous considerations, we are ready to estimate $\nabla D_{x}^{\alpha} \partial_{t}^{j} q$, where $|\alpha|+2 j \leq$ $2 k ;|\alpha|=\alpha_{1}+\alpha_{2}+\alpha_{3}, x=\left(x_{1}, x_{2}, x_{3}\right) ; D_{x}^{\alpha}=\frac{\partial^{|\alpha|}}{\partial_{x_{1}}^{\alpha_{1}} \partial_{x_{2}}^{\alpha_{2}} \partial_{x_{3}}^{\alpha_{3}}}$. Hence, it is seen that

$$
\left\|\nabla D_{x}^{\alpha} \partial_{t}^{j} q\right\|_{L_{p}\left(\mathcal{D}_{T}^{4}\right)} \leq c\left(\left\|b_{m}^{* *}\right\|_{W_{p}^{1-1 / p, 1 / 2-1 / 2 p}\left(\mathbb{R}_{+}^{3}\right)}+\left\|b_{3}^{* *}\right\|_{W_{p}^{2-1 / p, 1-1 / 2 p}\left(\mathbb{R}_{+}^{3}\right)}\right),
$$

where $b_{m}^{* *} \equiv D_{x^{\prime}}^{\alpha^{\prime}} \partial_{t}^{j} b_{m}$, for $\left|\alpha^{\prime}\right|+2 j \leq 2 k$ and from the assumption of the lemma,

$$
\begin{aligned}
& b_{m} \in W_{p}^{2 k+1-1 / p, k+1 / 2-1 / 2 p}\left(\mathbb{R}_{+}^{3}\right) \quad(m=1,2), \\
& b_{3} \in W_{p}^{2 k+2-1 / p, k+1-1 / 2 p}\left(\mathbb{R}_{+}^{3}\right) .
\end{aligned}
$$

These considerations imply that

$$
\|\nabla q\|_{W_{p}^{2 k, k}\left(\mathcal{D}_{T}^{4}\right)} \leq c\left(\left\|b_{m}\right\|_{W_{p}^{2 k+1-1 / p, k+1 / 2-1 / 2 p}\left(\mathbb{R}_{+}^{3}\right)}+\left\|b_{3}\right\|_{W_{p}^{2 k+2-1 / p, k+1-1 / 2 p}\left(\mathbb{R}_{+}^{3}\right)}\right) .
$$

In a similar way we show that

$$
\|v\|_{W_{p}^{2 k+2, k+1}\left(\mathcal{D}_{T}^{4}\right)} \leq c\left(\left\|b_{m}\right\|_{W_{p}^{2 k+1-1 / p, k+1 / 2-1 / 2 p}\left(\mathbb{R}_{+}^{3}\right)}+\left\|b_{3}\right\|_{W_{p}^{2 k+2-1 / p, k+1-1 / 2 p}\left(\mathbb{R}_{+}^{3}\right)}\right) .
$$

Next we have to consider the following problem on the whole space

$$
\begin{aligned}
& v_{, t}-\nu \Delta v+\nabla q=f, \\
& \operatorname{div} v=d, \\
& \left.v\right|_{t=0}=v_{0},
\end{aligned}
$$

for $f \in W_{p}^{2 k, k}\left(\mathbb{R}^{4}\right), d \in W_{p}^{2 k+1, k}\left(\mathbb{R}^{4}\right), v_{0} \in W_{p}^{2 k+2-2 / p}\left(\mathbb{R}^{3}\right)$. The trace theorem provides us with the existence of a function $\phi \in W_{p}^{2 k+2, k+1}\left(\mathbb{R}^{4}\right)$ such that $\left.\phi\right|_{t=0}=v_{0}$ and the following inequality holds

$$
\|\phi\|_{W_{p}^{2 k+2, k+1}\left(\mathbb{R}^{4}\right)} \leq c\left\|v_{0}\right\|_{W_{p}^{2 k+2-2 / p}\left(\mathbb{R}^{3}\right)} .
$$


We introduce a new function $\omega=v-\phi$, and consider the problem on $\omega$,

$$
\begin{aligned}
& \omega_{, t}-\nu \Delta \omega+\nabla q=f_{1} \in W_{p}^{2 k, k}\left(\mathbb{R}^{4}\right), \\
& \operatorname{div} \omega=d_{1} \in W_{p}^{2 k+1, k}\left(\mathbb{R}^{4}\right), \\
& \left.\omega\right|_{t=0}=0,
\end{aligned}
$$

where $d_{1}=d-\operatorname{div} \phi, f_{1}=f-\phi_{, t}+\nu \Delta \phi$.

Now, we consider the following problem

$$
\Delta \psi=d_{1} .
$$

We see that a solution of (3.14) exists and

$$
\|\nabla \psi\|_{W_{p}^{2 k+2, k}\left(\mathbb{R}^{4}\right)} \leq c\left\|d_{1}\right\|_{W_{p}^{2 k+1, k}\left(\mathbb{R}^{4}\right)} .
$$

If we take $\omega_{1}=\omega-\nabla \psi$ system (3.13) reduces to

$$
\begin{aligned}
& \omega_{1, t}-\nu \Delta \omega_{1}+\nabla q=f_{2}, \\
& \operatorname{div} \omega_{1}=0, \\
& \left.\omega_{1}\right|_{t=0}=0 .
\end{aligned}
$$

A solution to the above-mentioned problem has the explicit form (see [6]). We only need an estimate of $\nabla \partial_{x}^{\alpha} \partial_{t}^{j} q$ in the $L_{p}$-norm. Making use of the explicit form of the solution to the problem (3.15) we have $\nabla \partial_{x}^{\alpha} \partial_{t}^{j} q=\nabla \partial_{t}^{j} \tilde{q}$, where $\tilde{q}=\partial_{x}^{\alpha} q$ and $|\alpha|+2 j \leq 2 k ; \partial_{x}^{\alpha} q$ and $\partial_{x}^{\alpha} \omega_{1}$ are estimated from the problem (3.15) for all $|\alpha| \leq 2 k$.

Let us consider

$$
\nabla \partial_{t}^{j} \tilde{q}=\nabla \partial_{t}^{j} F_{t, x}^{-1}(\hat{\tilde{q}})
$$

where

$$
\hat{\tilde{q}}=\frac{i \xi_{1} \hat{\tilde{f}}_{2}^{1}+i \xi_{2} \hat{\tilde{f}}_{2}^{2}-i \xi_{3} \hat{\tilde{f}}_{2}^{3}}{|\xi|^{2}}
$$

and

$$
\hat{\tilde{f}}_{2}=\left(\hat{\tilde{f}}_{2}^{1}, \hat{\tilde{f}}_{2}^{2}, \hat{\tilde{f}}_{2}^{3}\right), \quad \tilde{f}_{2}=\partial_{x}^{\alpha} f_{2}
$$

This implies that

$$
\nabla \partial_{t}^{j} \tilde{q} \sim \frac{1}{(2 \pi)^{2}} \int_{R} e^{s t} \int_{R^{3}} e^{i x \cdot \xi} s^{j}\left(\hat{\tilde{f}}_{2}^{1}+\hat{\tilde{f}}_{2}^{2}-\hat{\tilde{f}}_{2}^{3}\right) d \xi_{0} d \xi
$$

and

$$
\left\|\nabla \partial_{t}^{j} \tilde{q}\right\|_{L_{p}\left(\mathbb{R}^{4}\right)} \leq c\left\|\partial_{t}^{j} \tilde{g}_{2}\right\|_{L_{p}\left(\mathbb{R}^{4}\right)} \leq c\left\|f_{2}\right\|_{W_{p}^{2 k, k}\left(\mathbb{R}^{4}\right)} .
$$

These considerations give the following inequalities

$$
\left\|\omega_{1}\right\|_{W_{p}^{2 k+2, k+1}\left(\mathbb{R}^{4}\right)}+\|\nabla q\|_{W_{p}^{2 k, k}\left(R^{4}\right)} \leq c\left\|f_{2}\right\|_{W_{p}^{2 k, k}\left(\mathbb{R}^{4}\right)} .
$$

Using representations of solutions to problem (3.12), $v=\omega_{1}+\nabla \psi+\phi$ we have the existence of $(v, \nabla q)$ such that $v \in W_{p, l o c}^{2 k+2, k+1}\left(\mathbb{R}^{4}\right), \nabla q \in W_{p, l o c}^{2 k, k}\left(\mathbb{R}^{4}\right)$ and the following inequality

$$
\begin{aligned}
& \|v\|_{W_{p}^{2 k+2, k+1}\left(\mathbb{R}^{4}\right)}+\|\nabla q\|_{W_{p}^{2 k, k}\left(\mathbb{R}^{4}\right)} \leq c\left(\|f\|_{W_{p}^{2 k, k}\left(\mathbb{R}^{4}\right)}\right. \\
& \left.+\|d\|_{W_{p}^{2 k+1, k}\left(\mathbb{R}^{4}\right)}+\|A\|_{W_{p}^{2 k, k}\left(\mathbb{R}^{4}\right)}+\|B\|_{W_{p}^{2 k, k}\left(\mathbb{R}^{4}\right)}+\left\|v_{0}\right\|_{W_{p}^{2 k+2-2 / p}\left(\mathbb{R}^{3}\right)}\right) .
\end{aligned}
$$


Therefore, using the considerations for the system (3.3) and (3.12) with estimates (3.11) and (3.16) and using [1] we conclude the proof of Lemma 3.1. Next we have to prove the existence in Besov spaces in a half space but with zero initial data $\left.u\right|_{t=0}=0$, since we need this result to prove Theorem 1 in a bounded domain by applying the regularizer technique. Obviously there exists a function $\tilde{v} \in W_{p}^{2 k+2, k+1}\left(\mathbb{R}_{+}^{4}\right)$ such as

$$
\left.\tilde{v}\right|_{t=0}=v_{0} \quad \text { and } \quad\|\tilde{v}\|_{W_{p}^{2 k+2, k+1}\left(\mathbb{R}_{+}^{4}\right)} \leq c\left\|v_{0}\right\|_{W_{p}^{2 k+2-2 / p}\left(\mathbb{R}_{+}^{3}\right)} .
$$

We assume that $\omega=v-\tilde{v}$. Then $\omega$ is a solution of the following homogeneous problem

$$
\begin{aligned}
& \omega_{, t}-\nu \Delta \omega+\nabla q=f-\tilde{v}_{, t}+\nu \Delta \tilde{v} \in W_{p}^{2 k, k}\left(\mathcal{D}_{T}^{4}\right) \\
& \operatorname{div} \omega=G-\operatorname{div} \tilde{v} \in \stackrel{\circ}{W}_{p}^{2 k+1, k}\left(\mathcal{D}_{T}^{4}\right) \\
& \omega_{i, x_{3}}+\left.\omega_{3, x_{i}}\right|_{x_{3}=0}=b_{i}-\bar{n} \cdot D(\tilde{v}) \cdot \bar{\tau}_{i} \in \stackrel{\circ}{W}_{p}^{2 k+1-1 / p, k+1 / 2-1 / p}\left(\mathbb{R}_{+}^{3}\right) \\
& \left.\omega_{3}\right|_{x_{3}=0}=b_{3}-\tilde{v}_{3} \in \stackrel{\circ}{W}_{p}^{2 k+2-1 / p, k+1-1 / 2 p}\left(\mathbb{R}_{+}^{3}\right) \\
& \left.\omega\right|_{t=0}=0
\end{aligned}
$$

where $\omega \in \stackrel{\circ}{W}{ }_{p}^{2 k+2, k+1}\left(\mathcal{D}_{T}^{4}\right)$ and the following estimate holds

$$
\begin{aligned}
& \|\omega\|_{W_{p}^{2 k+2, k+1}\left(\mathcal{D}_{T}^{4}\right)}+\|\nabla q\|_{W_{p}^{2 k, k}\left(\mathcal{D}_{T}^{4}\right)} \leq c\left(\|f\|_{W_{p}^{2 k, k}\left(\mathcal{D}_{T}^{4}\right)}+\|G\|_{W_{p}^{2 k+1, k}\left(\mathcal{D}_{T}^{4}\right)}\right. \\
& \quad+\left\|b_{i}\right\|_{W_{p}^{2 k+1-1 / p, k+1 / 2-1 / 2 p}\left(\mathbb{R}_{+}^{3}\right)}+\left\|b_{3}\right\|_{W_{p}^{2 k+2-1 / p, k+1-1 / 2 p}\left(\mathbb{R}_{+}^{3}\right)} \\
& \left.\quad+\|A\|_{W_{p}^{2 k, k}\left(\mathcal{D}_{T}^{4}\right)}+\|B\|_{W_{p}^{2 k, k}\left(\mathcal{D}_{T}^{4}\right)}\right) \quad \text { for } \quad k \in \mathbb{Z}_{+} .
\end{aligned}
$$

Using now Theorem 2.4, Lemma 3.1 we obtain the following result.

LEMMA 3.2. Let $\tilde{v} \in B_{p, q}^{2 s+2, s+1}\left(\mathbb{R}_{+}^{4}\right)$ be a function such that $\left.\tilde{v}\right|_{t=0}=v_{0}, v_{0} \in B_{p, q}^{2 s+2-2 / p}\left(\mathbb{R}_{+}^{3}\right)$ and $\|\tilde{v}\|_{B_{p, q}^{2 s+2, s+1}\left(\mathbb{R}_{+}^{4}\right)} \leq c\left\|v_{0}\right\|_{B_{p, q}^{2 s+2-2 / p}}\left(\mathbb{R}_{+}^{3}\right)$. Suppose that $f^{\prime}=f-\tilde{v}_{, t}+\nu \Delta \tilde{v}, G^{\prime}=$ $G-\operatorname{div} \tilde{v}, b_{i}^{\prime}=b_{i}-\left.\bar{n} \cdot \mathbb{D}(\tilde{v}) \cdot \bar{\tau}_{i}\right|_{x_{3}=0}, b_{3}^{\prime}=b_{3}-\left.\tilde{v}_{3}\right|_{x_{3}=0}, f^{\prime} \in B_{p, q}^{2 s, s}\left(\mathcal{D}_{T}^{4}\right), G^{\prime} \in \stackrel{\circ}{B}_{p, q}^{2 s+1, s}\left(\mathcal{D}_{T}^{4}\right)$, $b_{i}^{\prime} \in \stackrel{\circ}{B}_{p, q}^{2 s+1-1 / p, s+1 / 2-1 / 2 p}\left(\mathbb{R}_{+}^{3}\right), b_{3}^{\prime} \in \stackrel{\circ}{B}_{p, q}^{2 s+2-1 / p, s+1-k / p}\left(\mathbb{R}_{+}^{3}\right)$. Then problem $(3.18)$ has a unique solution such that $\omega \in \stackrel{\circ}{B}_{p, q}^{2 s+2, s+1}\left(\mathcal{D}_{T}^{4}\right)$ and $\nabla q \in B_{p, q}^{2 s, s}\left(\mathcal{D}_{T}^{4}\right)$ and

$$
\begin{aligned}
& \|\omega\|_{B_{p, q}^{2 s+2, s+1}\left(\mathcal{D}_{T}^{4}\right)}+\|\nabla q\|_{B_{p, q}^{2 s, s}\left(\mathcal{D}_{T}^{4}\right)} \leq c\left(\|f\|_{B_{p, q}^{2 s, s}\left(\mathcal{D}_{T}^{4}\right)}+\|A\|_{B_{p, q}^{2 s, s}\left(\mathcal{D}_{T}^{4}\right)}\right. \\
& \quad+\|B\|_{B_{p, q}^{2 s, s}\left(\mathcal{D}_{T}^{4}\right)}+\|G\|_{B_{p, q}^{2 s+1, s}\left(\mathcal{D}_{T}^{4}\right)}+\left\|v_{0}\right\|_{B_{p, q}^{2 s+2-2 / p}\left(\mathbb{R}_{+}^{3}\right)} \\
& \left.\quad+\left\|b_{i}\right\|_{B_{p, q}^{2 s+1-1 / p, s+1 / 2-1 / 2 p}\left(\mathbb{R}_{+}^{3}\right)}+\left\|b_{3}\right\|_{B_{p, q}^{2 s+2-1 / p, s+1-1 / 2 p}\left(\mathbb{R}_{+}^{3}\right)}\right) .
\end{aligned}
$$

Proof. One can see that assuming $A_{0}^{(1)}=L_{p}\left(\mathcal{D}_{T}^{4}\right), A_{0}^{(2)}=\stackrel{\circ}{W_{p}^{1,0}}\left(\mathcal{D}_{T}^{4}\right), A_{0}^{(3)}$ and $A_{0}^{(4)}=$ $\stackrel{\circ}{W}_{p}^{1-1 / p, 1 / 2-1 / 2 p}\left(\mathbb{R}_{+}^{3}\right), A_{0}^{(5)}=\stackrel{\circ}{W_{p}^{2-1 / p}, 1-1 / 2 p}\left(\mathbb{R}_{+}^{3}\right), A_{1}^{(1)}=W_{p}^{2 k, k}\left(\mathcal{D}_{T}^{4}\right), A_{1}^{(2)}=\stackrel{\circ}{W_{p}^{2 k+1, k}}\left(\mathcal{D}_{T}^{4}\right)$, $A_{1}^{(3)}$ and $A_{1}^{(3)}=\stackrel{\circ}{W}_{p}^{2 k+1-1 / p, k+1 / 2-1 / 2 p}\left(\mathbb{R}_{+}^{3}\right), A_{1}^{(5)}=W_{p}^{2 k+2-1 / p, k+1-1 / 2 p}\left(\mathbb{R}_{+}^{3}\right), B_{0}^{(1)}=$ $\stackrel{\circ}{W_{p}^{2,1}}\left(\mathcal{D}_{T}^{4}\right), B_{0}^{(2)}=L_{p}\left(\mathcal{D}_{T}^{4}\right), B_{1}^{(1)}=\stackrel{\circ}{W_{p}^{2 k+2, k+1}}\left(\mathcal{D}_{T}^{4}\right), B_{1}^{(2)}=W_{p}^{2 k, k}\left(\mathcal{D}_{T}^{4}\right)$, and denoting $A_{0}=\prod_{j=1}^{5} A_{0}^{(j)}, A_{1}=\prod_{j=1}^{5} A_{1}^{(j)}, B_{0}=\prod_{k=1}^{2} B_{0}^{(k)}, B_{1}=\prod_{k=1}^{2} B_{1}^{(k)}$ there exist well defined operators $T_{0}$ and $T_{1}$ such that $T_{0}: A_{0} \rightarrow B_{0}, T_{1}: A_{1} \rightarrow B_{1}$ which are linear and continuous if $a_{0}, a_{1}$ are elements of spaces $A_{0}$ and $A_{1}$. Moreover $T_{0}\left(a_{0}\right)=(v, \nabla q) \in$ $B_{0}, T_{1}\left(a_{1}\right)=(v, \nabla q) \in B_{1}$ satisfy estimate (3.19) which follows from Lemma 3.1 with 
homogeneous initial data conditions. Using interpolation $\left(A_{0}, A_{1}\right)_{\theta, q}$ and $\left(B_{0}, B_{1}\right)_{\theta, q}$ we have the existence of an operator $T$ such that $T:\left(A_{0}, A_{1}\right)_{\theta, q} \rightarrow\left(B_{0}, B_{1}\right)_{\theta, q}$, where $T$ is continuous and linear. Moreover we have

$$
\left(\stackrel{\circ}{W}_{p}^{2,1}, \stackrel{\circ}{W}_{p}^{2 k+2, k+1}\right)_{\theta, q}=\stackrel{\circ}{B}_{p q}^{2 s+2, s+1},
$$

where $s=(1-\theta)+\theta k, \theta \in[0,1]$.

These considerations conclude the proof of the lemma.

4. The problem in a bounded domain. To solve the problem (1.1) in a bounded domain $\Omega$ we have to define a partition of unity. We take two collections of open sets: $\left\{\omega^{(k)}\right\}$ and $\left\{\Omega^{(k)}\right\}$ such as $\bar{\omega}^{(k)} \subset \Omega^{(k)} \subset \Omega, \bigcup_{k} \omega^{(k)}=\Omega$ and $\bigcup_{k} \Omega^{(k)}=\Omega$ with $k \in$ $\mathcal{M} \cup N$. If $k \in M$ then $\bar{\Omega}^{(k)} \cap S=\varnothing$ and if $k \in N$ then $\bar{\omega}^{(k)} \cap S \neq \varnothing$.

We assume that $\sup _{k} \operatorname{diam} \Omega^{(k)} \leq 2 \lambda$ for some $\lambda$ small enough. Let $\zeta^{(k)}$ be a smooth function such that $0 \leq \zeta^{(k)} \leq 1$ and

$$
\zeta^{(k)}(x)= \begin{cases}1 & \text { for } x \in \omega^{(k)} \\ 0 & \text { for } x \in \Omega / \Omega^{(k)}\end{cases}
$$

and

$$
\left|D_{x}^{\nu} \zeta^{(k)}(x)\right| \leq \frac{c}{\lambda^{|\nu|}} \quad \text { and } \quad 1 \leq \sum_{k}\left(\left.\zeta^{(k)}(x)\right|^{2} \leq N_{0}\right.
$$

Let

$$
\Pi^{(k)}=\frac{\zeta^{(k)}}{\sum_{i}\left(\zeta^{(i)}\right)^{2}} .
$$

We see that $\Pi^{(k)}(x)=0$ for $x \in \Omega \backslash \Omega^{(k)},\left|D_{x}^{\nu} \Pi^{(k)}(x)\right| \leq \frac{c}{\lambda^{|\nu|}}$ and $\sum_{k} \Pi^{(k)}(x) \zeta^{(k)}(x)=1$.

We denote by $\xi^{(k)}$ a fixed point of $\omega^{(k)}$ for $k \in \mathcal{M}$, and of $\omega^{(k)} \cap S$ for $k \in N$. Let us consider a local coordinate system $y=\left(y_{1}, y_{2}, y_{3}\right)$ with the center at $\xi^{(k)}$. If $k \in N$ then the boundary part $\tilde{S}^{(k)}=S \cap \bar{\Omega}^{(k)}$ is described by $y_{3}=F\left(y_{1}, y_{2}\right)$. We choose the local system such as $F(0)=0, \nabla F(0)=0$.

From $S \in W_{p}^{2 s+3}$ we have $F \in W_{p}^{2 s+3-1 / p}$. We extend $F$ to $\bar{F}$ is such a way that $\bar{F}\left(y_{1}, y_{2}, 0\right)=F\left(y_{1}, y_{2}\right)$ and $\bar{F} \in W_{p}^{2 s+3}$ as well as $\bar{F} \in C^{2 s+1+\alpha}$ with $0<\alpha<2-3 / p$ and $\left|\nabla^{(2 s+1)}(\bar{F})\right| \leq c \lambda^{\alpha}$.

Now we can transform $\Omega^{(k)}$ into the half space by

$$
Z=\Phi_{k}(y)=(I d-\bar{F})(y) .
$$

Let $y=Y_{k}(x)$ be a transformation to the local coordinates $y$ which consists of translations and rotations. We consider the following problem in a bounded domain $\Omega$ :

$$
\begin{array}{ll}
v_{, t}-\nu \Delta v+\nabla p=f & \text { in } \Omega^{T}, \\
\operatorname{div} v=G & \text { in } \Omega^{T}, \\
\bar{n} \cdot D(v) \cdot \bar{\tau}_{i}+\left.\gamma v \cdot \bar{\tau}_{i}\right|_{s^{T}}=b_{i} \quad(i=1,2) & \text { on } S^{T}, \\
\left.v \cdot \bar{n}\right|_{s^{T}}=b_{3} & \text { on } S^{T}, \\
\left.v\right|_{t=0}=0 & \text { in } \Omega .
\end{array}
$$

Let us consider new variables $\tilde{v}=v \cdot \zeta^{(k)}, \tilde{p}=p \zeta^{(k)}, \tilde{f}=f \zeta^{(k)}, \tilde{G}=G \zeta^{(k)}, \tilde{b}_{i}=b_{i} \zeta^{(k)}$, $\tilde{b}_{3}=b_{3} \zeta^{(k)}$. Assume first that $k \in M$. Then we have problem (4.1) without boundary conditions after multiplying $(4.1)$ by $\zeta^{(k)}$. 
First we consider the following problem with $\zeta^{(k)} \equiv \zeta$

$$
\begin{aligned}
& \zeta v_{, t}-\nu \zeta \Delta v+\zeta \nabla q=\tilde{f}, \\
& \zeta \operatorname{div} v=\tilde{G}, \\
& \left.\tilde{v}\right|_{t=0}=0 .
\end{aligned}
$$

Now system (4.2) implies the following problem

$$
\begin{aligned}
& \tilde{v}_{, t}-\nu \Delta \tilde{v}+\nabla \tilde{q}=f_{1}, \\
& \operatorname{div} \tilde{v}=G_{1}, \\
& \left.\tilde{v}\right|_{t=0}=0,
\end{aligned}
$$

where $f_{1} \equiv \tilde{f}-2 \nu \nabla \zeta \cdot \nabla v-\nu v \Delta \zeta+p \nabla \zeta, G_{1}=\tilde{G}+v \cdot \nabla \zeta$.

Now, we obtain a condition on the new functions $f_{1}, G_{1}$ and compute functions $A_{\alpha, i}$, $B_{\alpha, i}$ satisfying the compatibility conditions

$$
\partial_{x}^{\alpha} \partial_{t}^{i+1} G_{1}-\operatorname{div} \partial_{x}^{\alpha} \partial_{t}^{i} f_{1}=\operatorname{div} \partial_{x}^{\alpha} \partial_{t}^{i} B+\partial_{x}^{\alpha} \partial_{t}^{i} A,
$$

where $A_{\alpha, i}=\partial_{x}^{\alpha} \partial_{t}^{i} A, B_{\alpha, i}=\partial_{x}^{\alpha} \partial_{t}^{i} B$. First we have to calculate the following terms $\partial_{x}^{\alpha} \partial_{t}^{i} G_{1}, \partial_{x}^{\alpha} \partial_{t}^{i} f_{1}$, where

$$
\partial_{x}^{\alpha} \partial_{t}^{i} G_{1}=\partial_{x}^{\alpha} \partial_{t}^{i}[v \cdot \nabla \zeta]+\partial_{x}^{\alpha} \partial_{t}^{i} \tilde{G}=\partial_{x}^{\alpha}\left(\zeta \partial_{t}^{i} G\right)+\partial_{x}^{\alpha}\left(\nabla \zeta \partial_{t}^{i} v\right)=I_{1}+I_{2}
$$

where $|\alpha|+2 i \leq[2 s]$

$$
I_{1}=\sum_{j=0}^{\alpha}\left(\begin{array}{c}
\alpha \\
j
\end{array}\right) \partial_{x}^{j} \zeta \partial_{x}^{\alpha-j} \partial_{t}^{j} G, \quad I_{2}=\sum_{j=0}^{\alpha}\left(\begin{array}{c}
\alpha \\
j
\end{array}\right) \nabla \partial_{x} \zeta \partial_{x}^{\alpha-j} \partial_{t}^{i} v .
$$

Next, we calculate

$$
\begin{aligned}
& \partial_{x}^{\alpha} \partial_{t}^{i} f_{1}=\partial_{x}^{\alpha} \partial_{t}^{i} \tilde{f}-2 \nu \partial_{x}^{\alpha} \partial_{t}^{i}(\nabla \zeta \cdot \nabla v)-\nu \partial_{x}^{\alpha} \partial_{t}^{i}(v \Delta \zeta)+\partial_{x}^{\alpha} \partial_{t}^{i}(q \nabla \zeta) \\
& =\sum_{j=0}^{\alpha}\left(\begin{array}{c}
\alpha \\
j
\end{array}\right) \partial_{x}^{j} \zeta \partial_{x}^{\alpha-j} \partial_{t}^{i} f-2 \nu \sum_{j=0}^{\alpha}\left(\begin{array}{c}
\alpha \\
j
\end{array}\right) \partial_{x}^{j} \nabla \zeta \partial_{x}^{\alpha-j} \partial_{t}^{i} \nabla v \\
& -\nu \sum_{j=0}^{\alpha}\left(\begin{array}{c}
\alpha \\
j
\end{array}\right) \partial_{x}^{j} \Delta \zeta \partial_{x}^{\alpha-j} \partial_{t}^{i} v+\sum_{j=0}^{\alpha}\left(\begin{array}{c}
\alpha \\
j
\end{array}\right) \partial_{x}^{j} \nabla \zeta \partial_{x}^{\alpha-j} \partial_{t}^{j} q
\end{aligned}
$$

which implies that

$$
\begin{aligned}
\partial_{x}^{\alpha} \partial_{t}^{i} f_{1}= & \sum_{j=0}^{\alpha}\left(\begin{array}{c}
\alpha \\
j
\end{array}\right) \partial_{x}^{j} \nabla \zeta\left(\partial_{x}^{\alpha-j} \partial_{t}^{i}\right)(q-2 \nu \nabla v) \\
& +\sum_{j=0}^{\alpha}\left(\begin{array}{c}
\alpha \\
j
\end{array}\right) \partial_{x}^{j} \zeta \partial_{x}^{\alpha-j} \partial_{t}^{i} q-\nu \sum_{j=0}^{\alpha}\left(\begin{array}{c}
\alpha \\
j
\end{array}\right) \Delta \partial_{x}^{j} \zeta \partial_{x}^{\alpha-j} \partial_{t}^{i} v .
\end{aligned}
$$

Then, we obtain

$$
I_{1}+I_{2}=\sum_{j=0}^{\alpha}\left(\begin{array}{l}
\alpha \\
j
\end{array}\right)\left[\partial_{x}^{j} \zeta \partial_{t}^{i} G+\nabla \partial_{x}^{j} \zeta \partial_{x}^{\alpha-j} \partial_{t}^{i} v\right]
$$

and

$$
\partial_{x}^{\alpha} \partial_{t}^{i} f_{1}=\sum_{j=0}^{\alpha}\left(\begin{array}{c}
\alpha \\
j
\end{array}\right)\left[\partial_{x}^{j} \nabla \zeta \partial_{x}^{\alpha-j} \partial_{t}^{i}(q-2 \nu \nabla v)+\partial_{x}^{j} \zeta \partial_{x}^{\alpha-j} \partial_{t}^{i} t-\nu \Delta \partial_{x}^{j} \zeta \partial_{x}^{\alpha-j} \partial_{t}^{i} v\right]
$$


Let us first assume that $|\alpha|+i=0$. Then on the basis of the compatibility conditions we get

$$
G_{1, t}-\operatorname{div} f_{1}=(\tilde{G}+v \cdot \nabla \zeta)_{, t}-\operatorname{div} \tilde{f}-\nabla \circ(q \nabla \zeta-2 \nu \nabla \zeta \cdot \nabla v-\nu v \Delta \zeta) .
$$

First we consider $\tilde{G}_{, t}+v_{, t} \cdot \nabla \zeta-\operatorname{div} \tilde{f}=J$, where $J$ is equal to

$$
\zeta\left(G_{, t}-\operatorname{div} f\right)-f \cdot \nabla \zeta+v_{, t} \cdot \nabla \zeta,
$$

From (4.1) and (4.7) we have

$$
\begin{aligned}
J & =\zeta(\operatorname{div} B+A)+\nabla \zeta(f-\nabla p+\nu \Delta v-f) \\
& =\zeta A+\operatorname{div}(\zeta B)-B \cdot \nabla \zeta+(\nu \Delta v-\nabla q) \cdot \nabla \zeta .
\end{aligned}
$$

Then we get

$$
J=\tilde{A}+\operatorname{div} \tilde{B}-\nabla \zeta \cdot B+\nabla \cdot(\nu \nabla \zeta \times \operatorname{rot} v-q \nabla \zeta)+q \Delta \zeta .
$$

Using (4.6) and (4.8) we obtain

$$
\begin{aligned}
G_{1, t}-\operatorname{div} f_{1}= & \tilde{A}-\nabla \zeta \cdot B+q \Delta \zeta+\nabla \cdot(\tilde{B}+\nu \nabla \zeta \times \operatorname{rot} v \\
& +2 \nu \nabla \zeta \cdot \nabla v+\nu v \Delta \zeta-2 q \nabla \zeta) .
\end{aligned}
$$

From (4.9) we can define new functions $A_{1}$ and $B_{1}$ by

$$
\begin{aligned}
& A_{1} \equiv \tilde{A}-\nabla \zeta \cdot B+q \Delta \zeta, \\
& B_{1} \equiv \tilde{B}+\nu \nabla \zeta \times \operatorname{rot} v+2 \nu \nabla \zeta \nabla v+\nu v \Delta \zeta-2 q \nabla \zeta,
\end{aligned}
$$

and the following compatibility condition holds

$$
G_{1, t}-\operatorname{div} f_{1}=\operatorname{div} B_{1}+A_{1},
$$

where $A_{1}, B_{1}$ are calculated explicitly. Let us put $G_{\alpha, i} \equiv \partial_{x}^{\alpha} \partial_{t}^{i} G_{1} ; G_{\alpha, i} \equiv \partial_{x}^{\alpha} \partial_{t}^{i} f_{1}, A_{\alpha, i}=$ $\partial_{x}^{\alpha} \partial_{t}^{i} A_{1}, B_{\alpha, i}=\partial_{x}^{\alpha} \partial_{t}^{i} B_{1}$. Then using (4.4)-(4.11) we have the compatibility conditions $\partial_{t} G_{\alpha, i}-\operatorname{div} F_{\alpha, i}=\operatorname{div} B_{\alpha, i}+A_{\alpha, i}$ where $B_{\alpha, i}$ and $A_{\alpha, i}$ are computed from (4.10). Then we have

$$
A_{\alpha, i}=\sum_{j=0}^{\alpha}\left(\begin{array}{c}
\alpha \\
j
\end{array}\right)\left[\partial_{x}^{j} \zeta \partial_{x}^{\alpha-j} \partial_{t}^{j} A-\nabla \partial_{x}^{j} \zeta \partial_{x}^{\alpha-j} \partial_{t}^{j} B+\partial_{x}^{j} \Delta \zeta \partial_{x}^{\alpha-j} \partial_{t}^{i} q\right]
$$

and

$$
\begin{gathered}
B_{\alpha, i}=\sum_{j=0}^{\alpha}\left(\begin{array}{c}
\alpha \\
j
\end{array}\right)\left[\partial_{x}^{j} \zeta \partial_{x}^{\alpha-j} \partial_{t}^{j} B+\nu \nabla \partial_{x}^{j} \zeta x \operatorname{rot} \partial_{x}^{\alpha-j} \partial_{t}^{j} v\right. \\
\left.+\nu \Delta \partial_{x}^{j} \zeta \partial_{x}^{\alpha-j} \partial_{t}^{i} v-\nabla \partial_{x}^{j} \zeta \partial_{x}^{\alpha-j} \partial_{t}^{i} q\right], \\
|j|=j_{1}+j_{2}+j_{3} .
\end{gathered}
$$

where $|\alpha|+2 i \leq[2 s]$ and $A_{1}, B_{1} \in B_{p, q}^{2 s, s}, A_{\alpha, i}$ and $B_{\alpha, i}$ belongs to $B_{p, q}^{2 s-\alpha-i, s-2 i-\alpha / 2}$.

Now in order to prove the existence of solutions for the problem (4.1) we need the following

LEMMA 4.1. Let $0<\varepsilon_{1}, \varepsilon_{2}<\frac{1}{2}$. Assume that $v \in B_{p, q}^{2 s+2, s+1}\left(\Omega^{T}\right)$ with $\left.v\right|_{t=0}=0$. Then the following inequality holds

$$
\|v\|_{B_{p, q}^{2 s+1, s+1 / 2}\left(\Omega^{T}\right)} \leq c\left(T^{1 / p}+T^{\chi}\right)\|v\|_{B_{p, q}^{2 s+2, s+1}\left(\Omega^{T}\right)},
$$


where $\chi=\left[s+1-\frac{\varepsilon_{2}}{2}\right]-\left[s+\frac{1}{2}+\frac{\varepsilon_{1}}{2}\right]+\frac{\varepsilon_{2}+\varepsilon_{1}}{2}-\frac{1}{2}>0, \Omega^{T}=\Omega \times(0, T)$ and $[n] \equiv \max \{k \in$ $\left.\mathbb{Z}_{+} \mid k \leq n\right\}$.

Proof. One can see that the following embeddings hold

$$
B_{p, q}^{2 s+2, s+1}\left(\Omega^{T}\right) \subset W_{p}^{2 s+2-\varepsilon_{2}, s+1-\frac{\varepsilon_{2}}{2}}\left(\Omega^{T}\right) \subset W_{p}^{2 s+1+\varepsilon_{1}, s+\frac{1}{2}+\frac{\varepsilon_{1}}{2}}\left(\Omega^{T}\right) \subset B_{p, q}^{2 s+1, s+1 / 2}\left(\Omega^{T}\right) .
$$

This implies the following inequality

$$
\|v\|_{W_{p}^{2 s+1+\varepsilon_{1}, s+1 / 2+\frac{\varepsilon_{1}}{2}}\left(\Omega^{T}\right)}=\left(\int_{0}^{T}\|v\|_{W_{p}^{2 s+1+\varepsilon_{1}}(\Omega)}^{p} d \tau+\int_{\Omega}\|v\|_{W_{p}^{s+1+\frac{\varepsilon_{1}}{2}}(0, T)}^{p} d x\right)^{1 / p},
$$

where

$$
\left(\int_{0}^{T}\|v\|_{W_{p}^{2 s+1+\varepsilon_{1}}(\Omega)} d x\right)^{1 / p} \leq T^{1 / p} \sup _{t}\|v\|_{W_{p}^{2 s+1+\varepsilon_{1}}(\Omega)} \leq c T^{1 / p}\|v\|_{B_{p, q}^{2 s+2, s+1}\left(\Omega^{T}\right)} .
$$

Next it is enough to consider the following integral

$$
\left(\int_{\Omega} \int_{0}^{T} \int_{0}^{T} \frac{\left|\partial_{t}^{j} v-\partial_{t^{\prime}}^{i} v\right|^{p}}{\left|t-t^{\prime}\right|^{1+p \sigma}} d x d t d t^{\prime}\right)^{1 / p} \equiv M,
$$

where $\sigma=s+\frac{1}{2}+\frac{\varepsilon_{1}}{2}-\left[s+\frac{1}{2}+\frac{\varepsilon_{1}}{2}\right]$. Then we obtain

$$
\begin{aligned}
M & =\left(\int_{\Omega} \int_{0}^{T} \int_{0}^{T} \frac{\left|\partial_{t}^{i} v-\partial_{t^{\prime}}^{i} v\right|^{P}}{\left|t-t^{\prime}\right|^{1+p \sigma}} \frac{\left|t-t^{\prime}\right|^{p \chi}}{\left|t-t^{\prime}\right|^{p \chi}} d x d t d t^{\prime}\right)^{1 / p} \\
& \leq T^{\chi}\left(\int_{\Omega} \int_{0}^{T} \int_{0}^{T} \frac{\left|\partial_{t}^{i} v-\partial_{t^{\prime}}^{i} v\right|^{P}}{\left|t-t^{\prime}\right|^{1+p \tilde{\sigma}}} d x d t d t^{\prime}\right)^{1 / p},
\end{aligned}
$$

where $\tilde{\sigma}=\sigma+\chi$ and $\chi$ are defined in the assumption of the lemma. This implies that

$$
M \leq C T^{\chi}\|v\|_{W_{p}^{2 s+2-\varepsilon_{2}, s+1-\varepsilon_{2} / 2 m}} \leq c T^{\chi}\|v\|_{B_{p, q}^{2 s+2, s+1}} .
$$

Adding (4.14) and (4.16) we obtain Lemma 4.1. This ends the proof.

Next, we have the following result

Lemma 4.2. Suppose that $S \in W_{p}^{2 s+3}, p \geq 2, f \in B_{p, q}^{2 s, s}\left(\Omega^{T}\right), G \in \stackrel{\circ}{B_{p, q}^{2 s+1, s}}\left(\Omega^{T}\right), b_{i} \in$ $\stackrel{\circ}{B}_{p, q}^{2 s+1-1 / p, s+1 / 2-1 / 2 p}\left(S^{T}\right)(i=1,2), b_{3} \in \stackrel{\circ}{B}_{p, q}^{2 s+2-1 / p, s+1-1 / 2 p}\left(S^{T}\right)$. Then for system 4.1 in a bounded domain we have a unique solution $(v, p)$ such that $v \in \stackrel{\circ}{B_{p, q}^{2 s+2, s+1}}\left(\Omega^{T}\right)$, $\nabla q \in B_{p, q}^{2 s, s}\left(\Omega^{T}\right)$, where the following compatibility conditions hold

$$
\partial_{t} F_{\alpha, i}-\operatorname{div} F_{\alpha, i}=\operatorname{div} B_{\alpha, i}+A_{\alpha, i} \quad \text { for } \quad|\alpha|+2 i \leq[2 s],
$$

where $A, B \in B_{p, q}^{2 s, s}\left(\Omega^{T}\right)$ and $\psi_{\alpha, i} \equiv \partial_{x}^{\alpha} \partial_{t}^{i} \psi$ for $|\alpha|+2 i \leq[2 s]$. Moreover the following estimates hold

$$
\begin{aligned}
\|v\|_{B_{p, q}^{2 s+2, s+1}}+\|\nabla q\|_{B_{p, q}^{2 s, s}\left(\Omega^{T}\right)} & \leq c\left(\|f\|_{B_{p, q}^{2 s, s}\left(\Omega^{T}\right)}+\|A\|_{B_{p, q}^{2 s, s}\left(\Omega^{T}\right)}+\|B\|_{p, q}^{2 s, s}\left(\Omega^{T}\right)\right. \\
& \left.+\left\|b_{i}\right\|_{B_{p, q}^{2 s+1-1 / p, s+1 / 2-1 / 2 p}\left(S^{T}\right)}+\left\|b_{3}\right\|_{B_{p, q}^{2 s+2-1 / p, s+1-1 / 2 p}\left(S^{T}\right)}\right)
\end{aligned}
$$

where

$$
\left\|B_{\alpha, i}\right\|_{B_{p, q}^{2 s-\alpha, s-2 i}} \leq c\left(\|B\|_{B_{p, q}^{2 s, s}}+\|A\|_{B_{p, q}^{2 s, s}}+\gamma_{1}\right)
$$

and

$$
\left\|A_{\alpha, i}\right\|_{B_{p, q}^{2 s-\alpha, s-2 i}} \leq c\left(\|B\|_{B_{p, q}^{2 s, s}}+\|A\|_{B_{p, q}^{2 s, s}}+\gamma_{2}\right),
$$

where $\gamma_{i}$ are defined in (4.18). 
Proof. Using (4.2)-(4.12) and Lemma 4.1 we can estimate $A_{\alpha, i}$ and $B_{\alpha, i}$ in Besov spaces. It can be shown that

$$
\begin{aligned}
& \left\|A_{\alpha, i}\right\|_{B_{p, q}^{2 s-\alpha, s-2 i}} \leq \sum_{j=0}^{\alpha}\left(\begin{array}{c}
\alpha \\
j
\end{array}\right) \frac{c}{\lambda^{|j|}}\left\|\partial_{x}^{\alpha-j} \partial_{t}^{i} A\right\|_{B_{p, q}^{2 s-\alpha, s-2 i}} \\
& \quad+\sum_{j=0}^{\alpha}\left(\begin{array}{l}
\alpha \\
j
\end{array}\right) \frac{c}{\lambda^{|j|+1}}\left\|\partial_{x}^{\alpha-j} \partial_{t}^{i} B\right\|_{B_{p, q}^{2 s-\alpha, s-2 i}} \\
& \quad+\sum_{j=0}^{\alpha}\left(\begin{array}{l}
\alpha \\
j
\end{array}\right) \frac{c}{\lambda^{|j|+2}}\left\|\partial_{x}^{\alpha-j} \partial_{t}^{j} q\right\|_{B_{p, q}^{2 s-\alpha, s-2 i}} ; \\
& \left\|B_{\alpha, i}\right\|_{B_{p, q}^{2 s-\alpha, s-2 i} \leq} \leq \sum_{j=0}^{\alpha}\left(\begin{array}{c}
\alpha \\
j
\end{array}\right) \frac{c}{\lambda^{|j|}}\left\|\partial_{x}^{\alpha-j} \partial_{t}^{j} B\right\|_{B_{p, q}^{2 s-\alpha, s-2 i}} \\
& \quad+\sum_{j=0}^{\alpha}\left(\begin{array}{l}
\alpha \\
j
\end{array}\right) \frac{c}{\lambda^{|j|+1}}\left\|\operatorname{rot} \partial_{x}^{\alpha-j} \partial_{t}^{i} v\right\|_{B_{p, q}^{2 s-\alpha, s-2 i}} \\
& \quad+\sum_{j=0}^{\alpha}\left(\begin{array}{l}
\alpha \\
j
\end{array}\right) \frac{c}{\lambda^{|j|+2}}\left\|\partial_{x}^{\alpha-j} \partial_{t}^{i} v\right\|_{B_{p, q}^{2 s-\alpha, s-2 i}} \\
& \quad+\sum_{j=0}^{\alpha}\left(\begin{array}{l}
\alpha \\
j
\end{array}\right) \frac{c}{\lambda^{|j|+1}}\left\|\partial_{x}^{\alpha-j} \partial_{t}^{i} q\right\|_{B_{p, q}^{2 s-\alpha, s-2 i}} .
\end{aligned}
$$

We also need the estimate for $A_{1}$ and $B_{1}$ in $B_{p, q}^{2 s, s}$.

Now using Lemma 3.2 to problem (4.3) we get

$$
\begin{gathered}
\|\tilde{v}\|_{B_{p, q}^{2 s+2, s+1}\left(D_{T}^{4}\right)}+\|\nabla \tilde{q}\|_{B_{p, q}^{2 s, s}} \leq c\left(\left\|f_{1}\right\|_{B_{p, q}^{2 s, s}\left(D_{T}^{4}\right)}+\left\|G_{1}\right\|_{B_{p, q}^{2 s+1, s}}+\left\|A_{1}\right\|_{B_{p, q}^{2 s, s}}\right. \\
+\left\|B_{1}\right\|_{B_{p, q}^{2 s, s}}+\sum_{\substack{|\alpha|+2 i=0 \\
|\alpha|, 2 i \geq 0}}^{[2 s]}\left(\left\|B_{\alpha, i}\right\|_{B_{p, q}^{2 s-\alpha, s-2 i}}+\left\|A_{\alpha, i}\right\|_{\left.B_{p, q}^{2 s-\alpha, s-2 i}\right)}\right)
\end{gathered}
$$

where

$$
\begin{aligned}
\left\|F_{1}\right\|_{B_{B_{p, q}^{2 s, s}}^{2 s,} \leq} & c\left(\|f\|_{B_{p, q}^{2 s, s}}+\frac{T^{1 / p}+T^{\chi}}{\lambda^{2 s+2}}\|v\|_{B_{p, q}^{2 s+2, s+1}}+\frac{1}{\lambda^{2 s+1}}\|q\|_{B_{p, q}^{2 s, s}}\right) \\
\left\|A_{1}\right\|_{B_{p, q}^{2 s, s}}+\left\|B_{1}\right\|_{B_{p, q}^{2 s, s}} \leq & c\left(\|A\|_{B_{p, q}^{2 s, s}}+\frac{c}{\lambda^{2 s+1}}\|B\|_{B_{p, q}^{2 s, s}}+\frac{c}{\lambda^{2 s+2}}\|q\|_{B_{p, q}^{2 s, s}}\right. \\
& \left.+\frac{1}{\lambda^{2 s+1}}\|v\|_{B_{p, q}^{2 s+1, s}}+\frac{1}{\lambda^{2 s+2}}\|v\|_{B_{p, q}^{2 s, s}}+\frac{1}{\lambda^{2 s+1}}\|q\|_{B_{p, q}^{2 s, s}}\right) .
\end{aligned}
$$

One can see that (4.18) can be estimated by r.h.s. of (4.21). Choosing $\lambda$ sufficiently small we need to find an estimate of the r.h.s. of (4.21). In order to do it we have to consider the following problem on pressure $q$ :

$$
\begin{aligned}
& \Delta q=0 \\
& \left.\frac{\partial q}{\partial n}\right|_{S}=g \quad \text { where } g=\nu \phi-b_{3, t} \text { and } \\
& \phi=\alpha_{1} v_{n}+\alpha_{2} v_{\tau_{i}, \tau_{i}}+\alpha_{3} v_{z_{i}}+\alpha_{4} v_{z_{i}, \tau_{i}}+\alpha_{5} b_{i, \tau_{i}}+\alpha_{6} b_{3, \tau_{i} \tau_{i}} \\
& \quad+\gamma v+a_{\beta} b_{\beta, \tau_{i}}-a_{\beta} v \cdot \bar{n}_{, \tau_{i}} \quad \text { for } \quad(i=1,2)
\end{aligned}
$$


and $v=v_{n} \cdot \bar{n}+v_{i} \tau_{i}$ (see [1]). The system (4.22) implies that

$$
\|q\|_{B_{p, q}^{2 s+2,0}} \leq c\|v\|_{B_{p, q}^{2 s+1,0}} .
$$

Using Lemma 4.1 we obtain

$$
\frac{1}{\lambda^{2 s+1}}\|q\|_{B_{p, q}^{2 s, s}} \leq \frac{c}{\lambda^{2 s+1}}\left(T^{1 / p}+T^{\chi}\right)\|v\|_{B_{p, q}^{2 s+2, s+1}} .
$$

Choosing $T$ small enough we obtain the estimate

$$
\|\tilde{v}\|_{B_{p, q}^{2 s+2, s+1}}+\|\nabla \tilde{q}\|_{B_{p, q}^{2 s, s}} \leq c\left(\|f\|_{B_{p, q}^{2 s, s}}+\|G\|_{B_{p, q}^{2 s+1, s}}+\|B\|_{B_{p, q}^{2 s, s}}+\|A\|_{B_{p, q}^{2 s, s}}\right) .
$$

We would like to have a similar estimate for $k \in N$. We write system (4.1) in z-coordinates:

$$
\begin{aligned}
& \tilde{v}_{, t}-\nu \Delta_{\mathbf{z}} \tilde{v}+\nabla_{\mathbf{z}} \tilde{q}=f_{1}+L_{1}\left(\partial_{\mathbf{z}}-\nabla \bar{F} \cdot \partial_{\mathbf{z}}\right)(\tilde{v}, \tilde{q}) \\
& \quad-L_{1}\left(\partial_{\mathbf{z}}\right)(\tilde{v}, \tilde{q}) \equiv f_{2}, \\
& \operatorname{div}_{\mathbf{z}} \tilde{v}=G_{1}+\nabla \bar{F} \cdot \partial_{\mathbf{z}} \tilde{v} \equiv G_{2}, \\
& \bar{e}_{\mathbf{Z}_{3}} \cdot D(\tilde{v}) \cdot \bar{\tau}_{i}+\gamma \tilde{v} \cdot \bar{\tau}_{i}=\Phi\left(\bar{n}\left(\eta_{, i} v_{j}+\eta_{, j} v_{i}\right) \cdot \bar{\tau}_{i}\right) \\
& \quad+\nabla \bar{F} \cdot D(\tilde{v}) \equiv b_{i}^{*}, \\
& \tilde{v} \cdot \bar{e}_{\mathbf{Z}_{3}}=b_{3}^{*},
\end{aligned}
$$

where $L_{1}\left(\partial_{x}\right)(v, q)=v_{, t}-\nu \Delta_{x} v+\nabla_{x} q$.

We need to find new functions $A_{2}, B_{2}$ which satisfy the compatibility condition

$$
G_{2, t}-\operatorname{div} f_{2}=\operatorname{div} B_{2}+A_{2} .
$$

First we can see that

$$
G_{1, t}-\operatorname{div} f_{1}=\operatorname{div} B_{1}+A_{1}
$$

From $(4.26)_{2}$ we have

$$
\bar{F}_{i, j} \tilde{v}_{j, i}=\partial_{\mathbf{z}_{i}}\left(\bar{F}_{i, j} \tilde{v}_{j}\right)-\tilde{v}_{j} \bar{F}_{i, j i}
$$

In order to obtain the new $B_{2}$ we have to consider the following problem

$$
\begin{aligned}
& \Delta h=\tilde{v}_{j, t} \bar{F}_{i, j i}, \\
& \left.h\right|_{\mathbf{z}_{3}=0}=0, \\
& h \rightarrow 0 \quad \text { as } \quad \mathbf{z}_{3} \rightarrow \infty .
\end{aligned}
$$

We see that

$$
\|\nabla h\|_{B_{p, q}^{2 s+1}\left(\mathbb{R}_{+}^{3}\right)} \leq c\left\|\tilde{v}_{j, t} \bar{F}_{i, j i}\right\|_{B_{p, q}^{2 s}\left(\mathbb{R}_{+}^{3}\right)} \leq c \varepsilon\|\tilde{v}\|_{B_{p, q}^{2 s+2, s+1}(\mathbb{R})}+c(\varepsilon) \lambda^{\alpha}\left\|\tilde{v}_{, t}\right\|_{B_{p, q}^{2 s, s}(\mathbb{R})} .
$$

Then we define $A_{2}$ and $B_{2}$ by

$$
B_{2}=B_{1}-\left(L_{1}\left(\partial_{\mathbf{z}}-\nabla \bar{F} \cdot \partial_{\mathbf{Z}}\right)(\tilde{v}, \tilde{q})-L_{1}\left(\partial_{\mathbf{z}}\right)(\tilde{v}, \tilde{q})\right)+\bar{F}_{i, j} \tilde{v}_{j}-\nabla h,
$$

$A_{2}=A_{1}$. We also need estimates for components of $B_{2}$. Hence we examine

$$
\begin{aligned}
& \left\|L_{1}\left(\partial_{\mathbf{z}}-\nabla \bar{F} \cdot \partial_{\mathbf{z}}\right)(\tilde{v}, \tilde{q})-L_{1}\left(\partial_{\mathbf{z}}(\tilde{v}, \tilde{q})\right)\right\|_{B_{p, q}^{2 s, s}\left(\mathcal{D}_{T}^{4}\right)} \\
& \quad \leq c\left\|\left|\nabla^{2} \bar{F}\left\|\nabla \tilde{v}\left|\left\|_{B_{p, q}^{2 s, s}\left(\mathcal{D}_{T}^{4}\right)}+c\right\|\right| \nabla \bar{F}|\cdot| \nabla^{2} \tilde{v} \mid\right\|_{B_{p, q}^{2 s, s}\left(\mathcal{D}_{T}^{4}\right)}\right.\right. \\
& \quad+c\|\mid \nabla \bar{F}\| \tilde{q}\|\|_{B_{p, q}^{2 s, s}} \leq\left(\delta+c(\delta) T^{a}+\left(T^{1 / 2}+T^{\chi}\right)+\lambda^{\alpha}\right)\|v\|_{B_{p, q}^{2 s+2, s+1},}, \\
& \quad\left\|\bar{F}_{i, j} \tilde{v}_{j}\right\|_{B_{p, q}^{2 s, s}}+\|\nabla h\|_{B_{p, q}^{2 s, s}} \leq\left(\varepsilon+c(\varepsilon) \lambda^{\alpha}+\left(T^{1 / 2}+T^{\chi}\right)\right)\|\tilde{v}\|_{B_{p, q}^{2 s+2, s+1} .}
\end{aligned}
$$


Using (4.26)-(4.33) we obtain

$$
\begin{aligned}
& \left\|f_{2}\right\|_{B_{p}^{2 s, q}} \leq\left\|f_{1}\right\|_{B_{p, q}^{2 s, s}}+c \lambda^{\alpha}\|\nabla \tilde{q}\|_{B_{p, q}^{2 s, s}}+\left(\delta+c(\delta) T^{a}+\left(T^{1 / 2}+T^{\chi}\right)\right)\|\tilde{v}\|_{B_{p, q}^{2 s+2, s+1}}, \\
& \left\|G_{2}\right\|_{B_{p, q}^{2 s+1, s}} \leq\left\|G_{1}\right\|_{B_{p, q}^{2 s+1, s}}+\left(\varepsilon+c(\varepsilon) \lambda^{\alpha}+\left(T^{1 / 2}+T^{\chi}\right)\right)\|\tilde{v}\|_{B_{p, q}^{2 s+2, s+1}}, \\
& \left\|b_{i}^{*}\right\|_{B_{p, q}^{2 s+1-1 / p, s+1 / 2-1 / 2 p}} \leq c\left(\frac{T^{1 / 2}+T^{\chi}}{\lambda^{2 s+1}}+\lambda^{\alpha}\right)\|\tilde{v}\|_{B_{p, q}^{2 s+2, s+1}}, \\
& \left\|b_{3}^{*}\right\|_{B_{p, q}^{2 s+2-1 / p, s+1-1 / 2 p}} \leq\left(\frac{T^{1 / 2}+T^{\chi}}{\lambda^{2 s+2}}\right)\|\tilde{v}\|_{B_{p, q}^{2 s+2, s+1}} \\
& \left\|A_{1}\right\|_{B_{p, q}^{2 s, s}} \leq\left\|A_{1}\right\|_{B_{p, q}^{2 s, s}}, \\
& \left\|B_{2}\right\|_{B_{p, q}^{2 s, s}} \leq\left\|B_{1}\right\|_{B_{p, q}^{2 s, s}}+\left(\delta+\varepsilon+c(\delta) T^{a}\right. \\
& \quad+\left((\varepsilon) \lambda^{\alpha}+\left(T^{1 / 2}+T^{\chi}\right)\right)\|\tilde{v}\|_{B_{p, q}^{2 s+2, s+1}}+\lambda^{\alpha}\|\nabla \tilde{q}\|_{B_{p, q}^{2 s, s}} .
\end{aligned}
$$

Using Lemma 3.2 and (4.34) we obtain

$$
\begin{aligned}
& \|\tilde{v}\|_{B_{p, q}^{2 s+2, s+1}}+\|\nabla \tilde{q}\|_{B_{p, q}^{2 s, s}} \leq c\left(\left\|f_{1}\right\|_{B_{p, q}^{2 s, s}}+\left\|G_{1}\right\|_{B_{p, q}^{2 s+1, s}}\right. \\
& +\left\|B_{1}\right\|_{B_{p, q}^{2 s, s}}+\left\|A_{1}\right\|_{B_{p, q}^{2 s, s}}+(\delta+c(\delta)) T^{a}+\varepsilon+c(\varepsilon) \lambda^{\alpha}+\left(T^{1 / 2}+T^{\chi}\right) \\
& \left.\quad+\frac{T^{1 / 2}+T^{\chi}}{\lambda^{2 s+1}}+\frac{T^{1 / 2}+T^{\chi}}{\lambda^{2 s+2}}+\lambda^{\alpha}\right)\left(\|\tilde{v}\|_{B_{p, q}^{2 s+2, s+1}}+\|\nabla \tilde{q}\|_{B_{p, q}^{2 s, s}}\right) .
\end{aligned}
$$

Let us define $d x=J_{\Phi^{-1}} d z$, where $J$ is the Jacobian matrix and $\left|J_{\Phi^{-1}}-I\right| \leq c \lambda^{\alpha}$. Thus, if we denote $\tilde{v}^{(\lambda)}=\zeta^{(\lambda)} v$ and $\tilde{q}^{(\lambda)}=\zeta^{(\lambda)} q$ we get

$$
\begin{aligned}
\|v\|_{B_{p, q}^{2 s+2, s+1}\left(\Omega^{T}\right)}+\|\nabla q\|_{B_{p, q}^{2 s, s}\left(\Omega^{T}\right)} & \\
& \leq \sum_{\lambda \in M \cup N}\left\|\tilde{v}^{(\lambda)}\right\|_{B_{p, q}^{2 s+2, s+1}\left(\Omega^{T}\right)}+\sum_{\lambda \in M \cup N}\left\|\nabla \tilde{q}^{(\lambda)}\right\|_{B_{p, q}^{2 s, s}\left(\Omega^{T}\right)} .
\end{aligned}
$$

Then, by (4.35) and properties of $\zeta^{(\lambda)}$ we obtain

$$
\begin{aligned}
& \|v\|_{B_{p, q}^{2 s+2, s+1}\left(\Omega^{T}\right)}+\|\nabla q\|_{B_{p, q}^{2 s, s}\left(\Omega^{T}\right)} \leq c N_{0}\left(\|f\|_{B_{p, q}^{2 s, s}\left(\Omega^{t}\right)}\right. \\
& \quad+\|B\|_{B_{p, q}^{2 s, s}\left(\Omega^{T}\right)}+\|A\|_{B_{p, q}^{2 s, s}\left(\Omega^{T}\right)}+\beta\left(\|v\|_{B_{p, q}^{2 s+2, s+1}\left(\Omega^{T}\right)}+\|\nabla q\|_{B_{p, q}^{2 s, s}\left(\Omega^{T}\right)}\right) \\
& \quad+\gamma\left(\|G\|_{B_{p, q}^{2 s+1, s}},\left\|b_{i}\right\|_{B_{p, q}^{2 s+1-1 / p, s+1 / 2-1 / 2 p}},\left\|b_{3}\right\|_{\left.\left.B_{p, q}^{2 s+2-1 / p, s+1-1 / 2 p}\right)\right) .}\right.
\end{aligned}
$$

where

$$
\beta \equiv \delta+c(\delta) T^{a}+\varepsilon+c(\varepsilon) \lambda^{\alpha}+\left(T^{1 / 2}+T^{\chi}\right)+\frac{T^{1 / 2}+T^{\chi}}{\lambda^{2 s+1}}+\frac{T^{1 / 2}+T^{\chi}}{\lambda^{2 s+2}}+\lambda^{\alpha} .
$$

We choose $\lambda$ and $T=T^{*}$ so small that $c N_{0} \beta<\frac{1}{2}$. Then we arrive at a solution $(v, q)$ such that $v \in \stackrel{\circ}{B}_{p, q}^{2 s+2, s+1}\left(\Omega^{T}\right), \nabla q \in B_{p, q}^{2 s, s}\left(\Omega^{T}\right)$ and

$$
\begin{aligned}
\|v\|_{B_{p, q}^{2 s+2, s+1}\left(\Omega^{T}\right)}+\|\nabla q\|_{B_{p, q}^{2 s, s}\left(\Omega^{T}\right)} & \\
& \leq c\left(\|f\|_{B_{p, q}^{2 s, s}\left(\Omega^{T}\right)}+\|B\|_{B_{p, q}^{2 s, s}\left(\Omega^{T}\right)}+\|A\|_{B_{p, q}^{2 s, s}\left(\Omega^{T}\right)}+\gamma\right) .
\end{aligned}
$$

where

(4.39) $\gamma \equiv\|G\|_{B_{p, q}^{2 s+1, s}\left(\Omega^{T}\right)}+N_{0}\left(\left\|b_{i}\right\|_{B_{p, q}^{2 s+1-1 / p, s+1 / 2-1 / 2 p}\left(S^{T}\right)}+\left\|b_{3}\right\|_{B_{p, q}^{2 s+2-1 / p, s+1-1 / 2 p}\left(S^{T}\right)}\right)$.

It finishes the proof of Lemma 4.2. 
5. The proof of Theorem 1. Our aim is to show the existence of solution to the problem (1.1),

$$
\begin{array}{ll}
v_{, t}-\nu \Delta v+\nabla q=f & \text { in } \Omega^{T}, \\
\operatorname{div} G=G & \text { in } \Omega^{T}, \\
\bar{n} \cdot D(v) \cdot \bar{\tau}_{i}+\left.\gamma v \cdot \bar{\tau}_{i}\right|_{s^{T}}=b_{i} \quad(i=1,2) & \text { on } S^{T}, \\
\left.v \cdot \bar{n}\right|_{S^{T}}=b_{3} & \text { on } S^{T}, \\
\left.v\right|_{t=0}=v_{0} & \text { in } \Omega .
\end{array}
$$

where the assumptions of Theorem 1 hold. From the theorems on traces we have the existence of function $\bar{u}_{0} \in B_{p, q}^{2 s+2, s+1}\left(\Omega^{T}\right)$ such as $\bar{u}_{0}(x, 0)=v_{0}(x)$ and $\left\|\bar{u}_{0}\right\|_{B_{p, q}^{2 s+2, s+1}\left(\Omega^{T}\right)} \leq$ $c\left\|v_{0}\right\|_{B_{p, q}^{2 s+2-2 / p}(\Omega)}$. Assuming that a solution of (1.1) has the form $v=w+\bar{u}_{0}$ problem (1.1) reduces to

$$
\begin{aligned}
& w_{, t}-\nu \Delta w+\nabla p=F_{1}, \\
& \operatorname{div} w=G_{1} \\
& \bar{n} \cdot D(w) \cdot \bar{\tau}_{i}+\left.\gamma w \cdot \bar{\tau}_{i}\right|_{s^{T}}=b_{i 1} \quad(i=1,2), \\
& \left.w \cdot \bar{n}\right|_{s^{T}}=b_{31} \\
& \left.w\right|_{t=0}=0
\end{aligned}
$$

where

$$
\begin{aligned}
& F_{1}=f-\bar{u}_{0, t}+\nu \Delta \bar{u}_{0}, \\
& G_{1}=G-\operatorname{div} \bar{u}_{0}, \\
& b_{i 1}=b_{i}-\bar{n} \cdot D\left(\bar{u}_{0}\right) \cdot \bar{\tau}_{i}-\gamma \bar{u}_{0} \cdot \bar{\tau}_{i} \quad(i=1,2), \\
& b_{31}=b_{3}-\bar{u}_{0} \cdot \bar{n},
\end{aligned}
$$

and the following compatibility condition holds

(5.4) $G_{1, t}-\operatorname{div} F_{1}=G_{, t}-\operatorname{div} f-\operatorname{div} \bar{u}_{0, t}+\operatorname{div} \bar{u}_{0, t}-\nu \operatorname{div} \Delta \bar{u}_{0}=\operatorname{div} B+A-\nu \operatorname{div} \Delta \bar{u}_{0}$.

Then, we have the existence of functions $A_{1}, B_{1}$ such that

$$
A_{1}=A, \quad B_{1}=B-\nu \Delta \bar{u}_{0} \quad \text { where } \quad G_{1, t}-\operatorname{div} F_{1}=\operatorname{div} B_{1}+A_{1} ;
$$

where $G_{1} \in \stackrel{\circ}{B}_{p, q}^{2 s+1, s}\left(\Omega^{T}\right),\left(B_{1} A_{1}, F_{1}\right) \in B_{p, q}^{2 s, s}\left(\Omega^{T}\right)$. Then using Lemma 4.2 we have the existence of the solution to problem (5.2) such that $w \in \stackrel{\circ}{B}_{p, q}^{2 s+2, s+1}\left(\Omega^{T}\right), \nabla q \in B_{p, q}^{2 s, s}\left(\Omega^{T}\right)$. Now using the representation $v=w+\bar{u}_{0}$, where $\left.v\right|_{t=0}=v_{0}$, we obtain

$$
\begin{aligned}
\|v\|_{B_{p, q}^{2 s+2, s+1}\left(\Omega^{T}\right) \leq} & \|w\|_{B_{p, q}^{2 s+2, s+1}\left(\Omega^{T}\right)}++\left\|\bar{u}_{0}\right\|_{B_{p, q}^{2 s+2, s+1}\left(\Omega^{T}\right)} \\
\leq & c\left\|v_{0}\right\|_{B_{p, q}^{2 s+2-2 / p}(\Omega)}+\|f\|_{B_{p, q}^{2 s, s}\left(\Omega^{T}\right)}+\|G\|_{B_{p, q}^{2 s+1, s}\left(\Omega^{T}\right)}+\|A\|_{B_{p, q}^{2 s, s}\left(\Omega^{T}\right)} \\
& +\|B\|_{B_{p, q}^{2 s, s}\left(\Omega^{T}\right)}+\left\|b_{i}\right\|_{B_{p, q}^{2 s+1-1 / p, s+1 / 2+1 / 2 p}\left(S^{T}\right)} \\
& \left.-\left\|b_{3}\right\|_{B_{p, q}^{2 s+2-1 / p, s+1-1 / 2 p}\left(S^{T}\right)}+X\right)
\end{aligned}
$$

for

$$
X \equiv \sum_{\substack{i=1 \\|\alpha|=0}}^{[2 s]}\left\|\left.\partial_{t}^{i-1} \partial_{x}^{\alpha} f\right|_{t=0}\right\|_{B_{p, q}^{2-2 / p}(\Omega)}, \quad|\alpha|+2 i \leq[2 s]
$$


Then we have the existence of solutions of system (1.1) for $T \leq T_{0}$ where $T_{0}=T^{*}$ and it is a small constant (see (4.36)).

Now it is enough to continue the solution to the intervals $\left[T_{0}, 2 T_{0}\right],\left[2 T_{0}, 3 T_{0}\right]$, $\left[2 T_{0}, 3 T_{0}\right]$, which proves Theorem 1 .

6. Proof of Theorem 2. Let us consider problem (1.1) in a half space in the following form

$$
\begin{array}{ll}
v_{, t}-\nu \Delta v+\nabla q=f & \text { in } \mathcal{D}_{T}^{4}, \\
\operatorname{div} v=G & \text { in } \mathcal{D}_{T}^{4}, \\
v_{1, x_{3}}+\left.v_{3, x_{1}}\right|_{x_{3}=0}=b_{1} & \text { on } \mathbb{R}_{+}^{3}, \\
v_{2, x_{3}}+\left.v_{3, x_{2}}\right|_{x_{3}=0}=b_{2} & \text { on } \mathbb{R}_{+}^{3}, \\
\left.v_{3}\right|_{x_{3}=0}=b_{3} & \text { on } \mathbb{R}_{+}^{3}, \\
\left.v\right|_{t=0}=0 & \text { in } \mathbb{R}_{+}^{3},
\end{array}
$$

for $\mathcal{D}_{T}^{4}=\mathbb{R}_{+}^{3} \times(0, \infty)_{x_{3}} \times(0, \infty)_{T}$. In order to solve (6.1) we first consider the homogeneous problem in a half space containing only boundary conditions

$$
\begin{aligned}
& v_{, t}-\nu \Delta v+\nabla q=0, \\
& \operatorname{div} v=0, \\
& v_{1, x_{3}}+\left.v_{3, x_{1}}\right|_{x_{3}=0}=b_{1}, \\
& v_{2, x_{3}}+\left.v_{3, x_{2}}\right|_{x_{3}=0}=b_{2}, \\
& \left.v_{3}\right|_{x_{3}=0}=b_{3} .
\end{aligned}
$$

From [1] we have an explicit representation of a solution of (6.2) after Fourier transformation of the following form

$$
\hat{v}_{i}\left(\xi^{\prime}, x_{3}, s\right)=\int_{0}^{\infty} e^{-s t} \int_{R^{2}} e^{-i x^{\prime} \cdot \xi^{\prime}} v_{i}(x, t) d x^{\prime} d t
$$

for $x^{\prime}=\left(x_{1}, x_{2}\right), \xi^{\prime}=\left(\xi_{1}, \xi_{2}\right), s=i \xi_{0}$, where $b_{i}$ are extended by zero for $t<0$. We have the following representation if $\nu=1$ (for simplicity). If $b_{2}=b_{3} \equiv 0$ then

$$
\begin{aligned}
\hat{v}_{1} & =\frac{1}{r} \hat{b}_{1} e^{-r x_{3}}+\frac{\xi_{1}^{2}}{s}\left(\frac{1}{\left|\xi^{\prime}\right|}-\frac{1}{r}\right) \hat{b}_{1} e^{-r x_{3}}+\frac{\xi_{1}^{2}}{s} \frac{\left|\xi^{\prime}\right|-r}{\left|\xi^{\prime}\right|}\left(\frac{e^{-\left|\xi^{\prime}\right| x_{3}}-e^{-r x_{3}}}{\left|\xi^{\prime}\right|-r}\right) \hat{b}_{1} \\
\hat{v}_{2} & =\left(\frac{1}{\left|\xi^{\prime}\right|}-\frac{1}{r}\right) \frac{\xi_{1} \xi_{2}}{s} \hat{b}_{1} e^{-r x_{3}}+\frac{\xi_{1} \xi_{2}}{s} \frac{\left|\xi^{\prime}\right|-r}{\left|\xi^{\prime}\right|}\left(\frac{1}{\left|\xi^{\prime}\right|-r}\right)\left(e^{-\left|\xi^{\prime}\right| x_{3}}-e^{-r x_{3}}\right) \hat{b}_{1} \\
\hat{v}_{3} & =\frac{i \xi_{1}}{r^{2}} \hat{b}_{1} \bar{e}^{r x_{3}}+\left(\frac{1}{\left|\xi^{\prime}\right|^{2}}-\frac{1}{r^{2}}\right) \frac{i \xi_{1}}{s}\left|\xi^{\prime}\right|^{2} \hat{b}_{1} e^{-r x_{3}} \\
& +\frac{i \xi_{1}}{s}\left|\xi^{\prime}\right|^{2} \frac{\left|\xi^{\prime}\right|-r}{\left|\xi^{\prime}\right|^{2}}\left(\frac{e^{-\left|\xi^{\prime}\right| x_{3}}-e^{-r x_{3}}}{\left|\xi^{\prime}\right|-r}\right) \hat{b}_{1} \\
\hat{q}= & \frac{i \xi_{1}}{\left|\xi^{\prime}\right|} \hat{b}_{1} e^{-\left|\xi^{\prime}\right| x_{3}} .
\end{aligned}
$$


If $b_{1}=b_{3} \equiv 0$ then

$$
\begin{aligned}
\hat{v}_{1} & =\left(\frac{1}{\left|\xi^{\prime}\right|}-\frac{1}{r}\right) \frac{\xi_{1} \xi_{2}}{s} \hat{b}_{2} e^{-r x_{3}}+\frac{\xi_{1} \xi_{2}}{s} \frac{\left(\left|\xi^{\prime}\right|-r\right)}{\left|\xi^{\prime}\right|}\left(\frac{e^{-\left|\xi^{\prime}\right| x_{3}}-e^{-r x_{3}}}{\left|\xi^{\prime}\right|-r}\right) \hat{b}_{2}, \\
\hat{v}_{2} & =-\frac{1}{r} \hat{b}_{2} e^{-r x_{3}}+\frac{\xi_{2}^{2}}{s}\left(\frac{1}{\left|\xi^{\prime}\right|}-\frac{1}{r}\right) e^{-r x_{3}} \hat{b}_{2}+\frac{\xi_{2}^{2}}{s} \frac{\left|\xi^{\prime}\right|-r}{\left|\xi^{\prime}\right|}\left(\frac{e^{-\left|\xi^{\prime}\right| x_{3}}-e^{-r x_{3}}}{\left|\xi^{\prime}\right|-r}\right) \hat{b}_{2}, \\
(6.4) \quad \hat{v}_{3} & =\frac{i \xi_{2}}{r^{2}} \hat{b}_{2} e^{-r x_{3}}+\left(\frac{1}{\left|\xi^{\prime}\right|}-\frac{1}{r^{2}}\right) \frac{i \xi_{2}}{s}\left|\xi^{\prime}\right|^{2} \hat{b}_{2} e^{-r x_{3}} \\
& +\frac{i \xi_{2}\left|\xi^{\prime}\right|}{s} \frac{\left|\xi^{\prime}\right|-r}{\left|\xi^{\prime}\right|^{2}}\left(\frac{e^{-\left|\xi^{\prime}\right| x_{3}}-e^{-r x_{3}}}{\left|\xi^{\prime}\right|-r}\right) \hat{b}_{2}, \\
\hat{q} & =\frac{i \xi_{2}}{\left|\xi^{\prime}\right|} \hat{b}_{2} e^{-\left|\xi^{\prime}\right| x_{3}} .
\end{aligned}
$$

Finally if $b_{2}=b_{1} \equiv 0$ then

$$
\begin{aligned}
\hat{v}_{1} & =\frac{i \xi_{1}}{r} \hat{b}_{3} e^{-r x_{3}}+\left(\frac{1}{r}-\frac{1}{\left|\xi^{\prime}\right|}\right) \frac{i \xi_{1}\left(\left|\xi^{\prime}\right|^{2}+r^{2}\right.}{s} e^{-r x_{3}} \hat{b}_{3} \\
& -\frac{\left(\left|\xi^{\prime}\right|-r\right)}{|\xi|} \frac{i \xi_{1}\left(\left|\xi^{\prime}\right|^{2}+r^{2}\right)}{s}\left(\frac{e^{-\left|\xi^{\prime}\right|^{2} x_{3}}-e^{-r x_{3}}}{\left|\xi^{\prime}\right|-r}\right) \hat{b}_{3}, \\
\hat{v}_{2} & =\frac{i \xi_{2}}{r} \hat{b}_{3} e^{-r x_{3}}+\left(\frac{1}{r}-\frac{1}{\left|\xi^{\prime}\right|}\right) \frac{i \xi_{2}\left(\left|\xi^{\prime}\right|^{2}+r^{2}\right)}{s} \hat{b}_{3} e^{-r x_{3}} \\
& -\frac{\left(\left|\xi^{\prime}\right|-r\right)}{\left|\xi^{\prime}\right|} \frac{i \xi_{2}\left(\left|\xi^{\prime}\right|^{2}+r^{2}\right)}{s}\left(\frac{e^{-\left|\xi^{\prime}\right| x_{3}}-e^{-r x_{3}}}{\left|\xi^{\prime}\right|-r}\right) \hat{b}_{3}, \\
\hat{v}_{3} & =\frac{-\left|\xi^{\prime}\right|^{2}}{r^{2}} \hat{b}_{3} e^{-r x_{3}}+\left(\frac{1}{\left|\xi^{\prime}\right|^{2}}-\frac{1}{r^{2}}\right) \frac{\left|\xi^{\prime}\right|^{2}\left(\left|\xi^{\prime}\right|^{2}+r^{2}\right)}{s} \hat{b}_{3} e^{-r x_{3}} \\
& +\frac{\left(\left|\xi^{\prime}\right|-r\right)\left(\left|\xi^{\prime}\right|^{2}+r^{2}\right)}{s}\left(\frac{e^{-|\xi| x_{3}}-e^{-r x_{3}}}{\left|\xi^{\prime}\right|-r} \hat{b}_{3},\right. \\
\hat{q} & =\frac{\left(\left|\xi^{\prime}\right|^{2}+r^{2}\right)}{\left|\xi^{\prime}\right|} \hat{b}_{3} e^{-\left|\xi^{\prime}\right| x_{3}},
\end{aligned}
$$

where $r^{2} \equiv\left|\xi^{\prime}\right|^{2}+s, s=i \xi_{0}$. We show the following

LEMma 6.1. Suppose that $b_{i} \in H^{2 s-3 / 2, s-3 / 4}\left(\mathbb{R}_{+}^{3}\right)$ for $(i=1,2) b_{3} \in H^{2 s-1 / 2, s-1 / 4}\left(\mathbb{R}_{+}^{3}\right)$, where $s \in\left(\frac{1}{2}, 1\right)$. Then system (6.2) has a unique solution $(v, q)$ such that $v \in H^{2 s, s}\left(\mathcal{D}_{T}^{4}\right)$, $q \in M_{2}^{2 s-1}\left(\mathcal{D}_{T}^{4}\right)$ and the following inequality holds

(6.6) $\|v\|_{H^{2 s, s}\left(\mathcal{D}_{T}^{4}\right)}+\|q\|_{M_{2}^{2 s-1}\left(D_{T}^{4}\right)} \leq c\left(\left\|b_{i}\right\|_{H^{2 s-3 / 2, s-3 / 4}\left(\mathbb{R}_{+}^{3}\right)}+\left\|b_{3}\right\|_{H^{2 s-1 / 2, s-1 / 4}\left(\mathbb{R}_{+}^{3}\right)}\right)$.

Proof. One can see the equivalence between

and $|(v, q)|_{X}$ where

$$
\|v\|_{H^{2 s, s}\left(\mathcal{D}_{T}^{4}\right)}+|q|_{M_{2}^{2 s-1}\left(\mathcal{D}_{T}^{4}\right)}
$$

$$
\begin{aligned}
|(v, q)|_{X}^{2}= & \int_{\mathbb{R}_{+}} \int_{\mathbb{R}_{+}^{3}}\left(\left(1+\left|\xi^{\prime}\right|^{s-2}\right)^{2}+\left(1+\left|\xi_{0}\right|^{s / 2}\right)^{2}\right)^{2}|\hat{v}|^{2} d \xi^{\prime} d \xi_{0} d x_{3} \\
& +\int_{\mathbb{R}_{+}} \int_{\mathbb{R}_{+}^{3}}\left(\left|\xi^{\prime}\right|^{2 s-1}|\hat{q}|\right)^{2} d \xi^{\prime} d \xi_{0} d x_{3} .
\end{aligned}
$$


In this paper we consider only estimates of $v_{11}$ and $q$ under the assumption $b_{2}=b_{3}=0$ and for the pressure $q$ with assumption $b_{1}=b_{2}=0$, where $\hat{v}_{11}=\frac{1}{r} \hat{b}_{1} e^{-r x_{3}}$ is the first term of $\hat{v}_{1}$ and

$$
v_{11}=\frac{1}{(2 \pi)^{3 / 2}} \int_{0}^{\infty} e^{s t} \int_{R_{+}^{2}} e^{i x^{\prime} \xi^{\prime}} \hat{v}_{11} d \xi^{\prime} d \xi_{0} .
$$

We get the estimates in a similar way. From equivalence (6.7) there exists a constant $c>0$ such that the following inequality holds

$$
\left\|v_{11}\right\|_{H^{2 s, s}\left(\mathcal{D}_{T}^{4}\right)}^{2}+|q|_{M_{2}^{2 s-1}\left(\mathcal{D}_{T}^{4}\right)}^{2} \leq c\left|\left(v_{11}, q\right)\right|_{X}^{2} .
$$

Then we obtain

$$
\begin{aligned}
\left|\left(v_{11}, q\right)\right|_{X}^{2}= & \int_{\mathbb{R}_{+}} \int_{\mathbb{R}_{+}^{3}}\left(\left(1+\left|\xi^{\prime}\right|^{2 s}\right)^{2}+\left(1+\left|\xi_{0}\right|^{s}\right)^{2}\right)\left|\frac{\hat{b}_{1}}{r} e^{-r x_{3}}\right|^{2} d \xi^{\prime} d \xi_{0} d x 3 \\
& +\int_{\mathbb{R}_{+}} \int_{\mathbb{R}_{+}^{3}}\left(\left|\xi^{\prime}\right|^{2 s-1}\left|\frac{i \xi_{1}}{\left|\xi^{\prime}\right|} \hat{b}_{1} e^{-\left|\xi^{\prime}\right| x_{3}}\right|\right)^{2} d \xi^{\prime} d \xi_{0} d x_{3} .
\end{aligned}
$$

Since $\frac{1}{r}=\frac{1}{\sqrt{\xi_{0}+\left|\xi^{\prime}\right|^{2}}}$ and $\xi_{0} \geq 0$ we have

$$
\begin{aligned}
\left|\left(v_{11}, q\right)\right|_{X}^{2} \leq & c \int_{\mathbb{R}_{+}} \int_{\mathbb{R}^{2}}\left(\left|\xi^{\prime}\right|^{4 s-2}+\left|\xi_{0}\right|^{2 s-1}+\frac{1}{\left|\xi^{\prime}\right|}+\frac{1}{\left|\xi_{0}\right|^{1 / 2}}\right)\left|\hat{b}_{1}\right|^{2}\left(\int_{0}^{\infty} e^{-2 r x_{3}} d x_{3}\right) d \xi \\
& +\int_{\mathbb{R}_{+}} \int_{\mathbb{R}^{2}}\left|\xi^{\prime}\right|^{4 s-2}\left|\hat{b}_{1}\right|^{2}\left(\int_{0}^{\infty} e^{-2\left|\xi^{\prime}\right| x_{3}} d x_{3}\right) d \xi_{0} d \xi^{\prime} .
\end{aligned}
$$

Then (6.9) implies that

$$
\left|\left(v_{11}, q\right)\right|_{X} \leq c\left\|b_{1}\right\|_{H^{2 s-3 / 2, s-3 / 4}\left(\mathbb{R}_{+}^{3}\right)}
$$

which holds from the Plancherel theorem. Now we estimate $(6.5)_{4}$ in the following form $\hat{q}=\frac{\left(\left|\xi^{\prime}\right|^{2}+r^{2}\right)}{\left|\xi^{\prime}\right|} \hat{b}_{3} e^{-\left|\xi^{\prime}\right| x_{3}}$. Then we get

$$
\begin{aligned}
& \int_{\mathbb{R}_{+}} \int_{\mathbb{R}_{+}^{3}}\left(\left|\xi^{\prime}\right|^{2 s-1}|\hat{q}|\right)^{2} d \xi_{0} d \xi^{\prime} d x_{3} \leq c \int_{\mathbb{R}_{+}} \int_{\mathbb{R}^{2}}\left(\left|\xi^{\prime}\right|^{4 s-2}\left|\xi^{\prime}\right|^{2}\left|\hat{b}_{3}\right|^{2} \int_{0}^{\infty} e^{-2\left|\xi^{\prime}\right| x_{3}} d x_{3}\right) d \xi_{0} d \xi^{\prime} \\
& \leq c \int_{\mathbb{R}_{+}^{3}}\left|\xi^{\prime}\right|^{4 s-1}\left|\hat{b}_{3}\right|^{2} d \xi=c \int_{\mathbb{R}_{+}^{3}}\left(\left|\xi^{\prime}\right|^{2 s-1 / 2}\left|\hat{b}_{3}\right|\right)^{2} d \xi \leq c\left\|b_{3}\right\|_{H^{2 s-1 / 2, s-1 / 4}\left(\mathbb{R}_{+}^{3}\right)}^{2} .
\end{aligned}
$$

These considerations conclude the proof of the lemma.

REMARK 1. $M_{2}^{2 s-1}\left(\mathcal{D}_{T}^{4}\right)$ is not a Banach space. We introduce $\tilde{M}_{2}^{2 s-1}\left(\mathcal{D}_{T}^{4}\right)=\{\phi: \phi \in$ $\left.M_{2}^{2 s-1}\left(\mathcal{D}_{T}^{4}\right) \mid \int_{\mathbb{R}_{+}^{3}} \phi d x=0\right\}$. Then the space $\tilde{M}_{2}^{2 s-1}\left(\mathcal{D}_{T}^{4}\right)$ is a Banach space.

REMARK 2. Let the assumptions of Lemma 6.1 hold. Suppose that $\int_{\mathbb{R}_{+}^{3}} q d x=0$. Then, there exists a unique solution to the problem (6.2) such that $v \in H^{2 s, s}\left(\mathcal{D}_{T}^{4}\right)$ and pressure $q \in \tilde{M}_{2}^{2 s-1}\left(\mathcal{D}_{T}^{4}\right)$ with the following estimate

$$
\|v\|_{H^{2 s, s}\left(\mathcal{D}_{T}^{4}\right)}+\|q\|_{\tilde{M}_{2}^{2 s-1}\left(\mathcal{D}_{T}^{4}\right)} \leq c\left(\left\|b_{i}\right\|_{H^{2 s-3 / 2, s-3 / 4}\left(\mathbb{R}_{+}^{3}\right)}+\left\|b_{3}\right\|_{H^{2 s-1 / 2, s-1 / 4}\left(\mathbb{R}_{+}^{3}\right)}\right) .
$$


Our next step is to show the existence of solutions in the whole space $\mathbb{R}^{4}$ of the following problem

$$
\begin{aligned}
& v_{, t}-\nu \Delta v+\nabla q=f, \\
& \operatorname{div} v=G \\
& \left.v\right|_{t=0}=0
\end{aligned}
$$

The following lemma holds:

Lemma 6.2. Let $f \in L_{2}\left(\mathbb{R}^{4}\right), G \in H^{1,0}\left(\mathbb{R}^{4}\right), \tilde{s} \in\left(\frac{1}{2}, 1\right), \int_{\mathbb{R}_{3}} q d x=0$ and the compatibility conditions hold. Then there exists a unique solution $(v, q)$ in the whole space such that $v \in H^{2 \tilde{s}, \tilde{s}}\left(\mathbb{R}^{4}\right), q \in \tilde{M}_{2}^{2 \tilde{s}-1}\left(\mathbb{R}^{4}\right)$ and the following estimate holds

$$
\|v\|_{H^{2 \tilde{s}, \tilde{s}\left(\mathbb{R}^{4}\right)}}+\|q\|_{\tilde{M}_{2}^{2 \tilde{s}-1}\left(\mathbb{R}^{4}\right)} \leq c\left(\|f\|_{L_{2}\left(\mathbb{R}^{4}\right)}+\lambda\|A\|_{L_{2}\left(\mathbb{R}^{4}\right)}+\|B\|_{L_{2}\left(\mathbb{R}^{4}\right)}+\|G\|_{H^{1,0}\left(\mathbb{R}^{4}\right)}\right) .
$$

where $A, B$ are functions such that

$$
G_{, t}-\operatorname{div} f=\operatorname{div} B+A,
$$

for supp $A<\lambda$.

Proof. Let us consider the following eliptic system

$$
\Delta \phi(x, t)=G(x, t) \text { in } \mathbb{R}^{4} .
$$

From $(6.13)_{3}$ and the representation of the solution (6.15) in the form

$$
\phi(x, t)=c \int_{\mathbb{R}^{3}} \frac{G(y, t)}{|x-y|} d y
$$

we have that the initial data $\left.\phi\right|_{t=0}$ equal to zero. Let us assume that the r.h.s. of (6.15) is in $H^{1,0}\left(\mathbb{R}^{4}\right)$.

Using the Fourier transform applied to system (6.15) we obtain

$$
\begin{aligned}
& |\xi|^{2} \hat{\phi}=\hat{G}(\xi, t), \quad \hat{G}(\xi, 0)=0 \text { where } \\
& \hat{\psi}(\xi, t) \equiv \frac{1}{(2 \pi)^{3 / 2}} \int_{\mathbb{R}^{3}} e^{-i x \cdot \xi} \psi(x, t) d x, \text { for } x \cdot \xi=\sum_{j=1}^{3} \xi_{j} x_{j}
\end{aligned}
$$

Then (6.16) implies that $\hat{\phi}(\xi, t)=\frac{\hat{F}(\xi, t)}{|\xi|^{2}}$.

Now we consider the following integral

$$
\int_{\mathbb{R}^{3}}\left(|\xi|^{3} \hat{\phi}\right)^{2} d \xi=\int_{\mathbb{R}^{3}}(|\xi| \hat{G})^{2} d \xi \leq c\|G\|_{H^{1}\left(\mathbb{R}^{3}\right)}^{2},
$$

Then using the Plancherel theorem we obtain

$$
\int_{\mathbb{R}}\|\phi\|_{H^{3}\left(\mathbb{R}^{3}\right)}^{2} d \tau \leq c \int_{\mathbb{R}}\|G\|_{H^{1}\left(\mathbb{R}^{3}\right)}^{2} d \tau \leq c\|G\|_{H^{1,0}\left(\mathbb{R}^{4}\right)}^{2} .
$$

This implies that if $G \in H^{1,0}\left(\mathbb{R}^{4}\right)$ then we get the following estimate $\|\nabla \phi\|_{H^{2,0}\left(\mathbb{R}^{4}\right)} \leq$ $c\|G\|_{H^{1,0}\left(\mathbb{R}^{4}\right)}$, where $\Delta \phi=G,\left.\phi\right|_{t=0}=0$. Let us represent solutions to (6.13) in the form 
$v \equiv w+\nabla \phi$. Then we obtain the problem on $w$,

$$
\begin{aligned}
& w_{, t}-\nu \Delta w+\nabla q=f^{1}, \\
& \operatorname{div} w=0 \\
& \left.w\right|_{t=0}=0
\end{aligned}
$$

where $f^{1} \equiv f-\nabla \phi_{, t}+\nu \Delta \nabla \phi$.

Let $\nabla \phi_{, t}=\nabla \phi_{, t}^{(1)}+\nabla \phi_{, t}^{(2)}$, then we get $\Delta \phi_{, t}^{(1)}=\operatorname{div}(F+B), \Delta \phi_{, t}^{(2)}=A$. One can see that this implies the following estimate

$$
\left\|\nabla \phi_{, t}^{(1)}\right\|_{L_{2}\left(\mathbb{R}^{4}\right)}+\left\|\nabla \phi_{, t}^{(2)}\right\|_{L_{2}\left(\mathbb{R}^{4}\right)} \leq c\left(\|f\|_{L_{2}\left(\mathbb{R}^{4}\right)}+\|B\|_{L_{2}\left(\mathbb{R}^{4}\right)}+\lambda\|A\|_{L_{2}\left(\mathbb{R}^{4}\right)}\right) .
$$

Adding (6.17) and (6.19) we obtain

$$
\begin{aligned}
\|\nabla \phi\|_{H^{2,1}\left(\mathbb{R}^{4}\right)} & \leq c\left(\|\nabla \phi\|_{H^{2,0}\left(\mathbb{R}^{4}\right)}+\|\nabla \phi\|_{H^{0,1}\left(\mathbb{R}^{4}\right)}\right) \\
& \leq c\left(\|G\|_{H^{1,0}\left(\mathbb{R}^{4}\right)}+\sqrt{\left.\int_{\mathbb{R}^{4}}|\nabla \phi|^{2} d x d \tau+\left\|\nabla \phi_{, t}\right\|_{L_{2}\left(\mathbb{R}^{4}\right)}^{2}\right)}\right. \\
& \leq c\left(\|G\|_{H^{1,0}\left(\mathbb{R}^{4}\right)}+\|f\|_{L_{2}\left(\mathbb{R}^{4}\right)}+\|B\|_{L_{2}\left(\mathbb{R}^{4}\right)}+\lambda\|A\|_{L_{2}\left(\mathbb{R}^{4}\right)}\right) .
\end{aligned}
$$

Using (6.18) and (6.20) we have

$$
\left\|f^{1}\right\|_{L_{2}\left(\mathbb{R}^{4}\right)} \leq c\left(\|f\|_{L_{2}\left(\mathbb{R}^{4}\right)}+\|G\|_{H^{1,0}\left(\mathbb{R}^{4}\right)}+\lambda\|A\|_{L_{2}\left(\mathbb{R}^{4}\right)}+\|B\|_{L_{2}\left(\mathbb{R}^{4}\right)}\right) .
$$

Now it is enough to solve the system (6.18). Applying the Fourier transform to the problem (6.18) we obtain the following equations

$$
\begin{aligned}
& s \hat{u}+\nu|\xi|^{2} \hat{u}+i \xi \hat{q}=\hat{f}^{1}, \\
& \sum_{j=1}^{3} \xi_{j} \hat{u}_{j}=0, \\
& \left.u\right|_{t=0}=0 .
\end{aligned}
$$

Let $r^{2}=|\xi|^{2}+s$. Then equations (6.22) can be expressed in the matrix form $A X=F$, where

$$
A=\left(\begin{array}{cccc}
r^{2} & 0 & 0 & i \xi_{1} \\
0 & r^{2} & 0 & i \xi_{2} \\
0 & 0 & r^{2} & i \xi_{3} \\
\xi_{1} & \xi_{2} & \xi_{3} & 0
\end{array}\right), \quad X=\left(\begin{array}{c}
\hat{u}_{1} \\
\hat{u}_{2} \\
\hat{u}_{3} \\
\hat{q}
\end{array}\right), \quad F=\left(\begin{array}{c}
\hat{f}_{1}^{1} \\
\hat{f}_{2}^{1} \\
\hat{f}_{3}^{1} \\
0
\end{array}\right)
$$

Solving this problem we have the solution $X$ in the following matrix form $X=A^{-1} F$, where

$$
A^{-1}=\left(\begin{array}{cccc}
\frac{\xi_{2}^{2}+\xi^{2}}{\nu r^{2}|\xi|^{2}} & -\frac{\xi_{1} \xi_{2}}{\nu r^{2}|\xi|^{2}} & -\frac{\xi_{1} \xi_{3}}{\nu r^{2}|\xi|^{2}} & \frac{\xi_{1}}{|\xi|^{2}} \\
\frac{-\xi_{1} \xi_{2}}{\nu r^{2}|\xi|^{2}} & \frac{\xi_{1}^{2}+\xi_{3}^{2}}{\nu r^{2}|\xi|^{2}} & \frac{-\xi_{2} \xi_{3}}{\nu r^{2}|\xi|^{2}} & \frac{\xi_{2}}{|\xi|^{2}} \\
\frac{-\xi_{1} \xi_{3}}{\nu r^{2}|\xi|^{2}} & \frac{-\xi_{2} \xi_{3}}{\nu r^{2}|\xi|^{2}} & \frac{\xi_{1}^{2}+\xi_{2}^{2}}{\nu r^{2}|\xi|^{2}} & \frac{\xi_{3}}{|\xi|^{2}} \\
\frac{-i \xi_{1}}{|\xi|^{2}} & \frac{i \xi_{2}}{|\xi|^{2}} & \frac{-i \xi_{3}}{|\xi|^{2}} & \frac{i \nu r^{2}}{|\xi|^{2}}
\end{array}\right) .
$$


From (6.24) we obtain

$$
\begin{aligned}
& \hat{u}_{1}=\frac{\left(\xi_{2}^{2}+\xi_{3}^{2}\right) \hat{f}_{1}^{1}-\xi_{1} \xi_{2} \hat{f}_{2}^{1}-\xi_{1} \xi_{3} \hat{f}_{3}^{1}}{\nu r^{2}|\xi|^{2}}, \\
& \hat{u}_{2}=\frac{-\xi_{1} \xi_{2} \hat{f}_{1}^{1}+\left(\xi_{1}^{2}+\xi_{3}^{2}\right) \hat{f}_{2}^{1}-\xi_{2} \xi_{3} \hat{f}_{3}^{1}}{\nu r^{2}|\xi|^{2}}, \\
& \hat{u}_{3}=\frac{-\xi_{1} \xi_{3} \hat{f}_{1}^{1}-\xi_{2} \xi_{3} \hat{f}_{2}^{1}+\left(\xi_{1}^{2}+\xi_{2}^{2}\right) \hat{f}_{3}^{1}}{\nu r^{2}|\xi|^{2}}, \\
& \hat{q}=-\frac{i \xi_{1} \hat{f}_{1}^{1}+i \xi_{2} \hat{f}_{2}^{1}+i \xi_{3} \hat{f}_{3}^{1}}{|\xi|^{2}} .
\end{aligned}
$$

From [5] with $p=2$ we have the existence of system (6.18) so that the velocity vector $w$ belongs to $H^{2,1}\left(\mathbb{R}^{4}\right)$ and $w$ is estimated by $f^{1}$ in $L_{2}\left(\mathbb{R}^{4}\right)$ norm. We only need to estimate pressure $q$ in $\tilde{M}_{2}^{2 \tilde{s}-1}\left(\mathbb{R}^{4}\right)$. One can see the following inequality

$$
\begin{aligned}
& \int_{\mathbb{R}^{4}}\left(|\xi|^{2 \tilde{s}-1}|\hat{q}|\right)^{2} d \xi d \xi_{0} \leq c \int_{\mathbb{R}^{4}}|\xi|^{4 \tilde{s}-2} \frac{\left|\hat{f}^{1}\right|^{2}}{|\xi|^{2}} d \xi d \xi_{0} \\
& \leq c \int_{\mathbb{R}^{4}}\left(|\xi|^{2 \tilde{s}-2}\left|\hat{f}^{1}\right|\right)^{2} d \xi d \xi_{0} \leq c\left\|f^{1}\right\|_{H^{2 \tilde{s}-2,0}\left(\mathbb{R}^{4}\right)} .
\end{aligned}
$$

for $\tilde{s} \in\left(\frac{1}{2}, 1\right)$. Now using the embedding $L_{2}\left(\mathbb{R}^{4}\right) \subset H^{2 \tilde{s}-2,0}\left(\mathbb{R}^{4}\right)$ for $2 \tilde{s} \leq 2$ we have that

$$
\|q\|_{\tilde{M}_{2}^{2 \tilde{s}-1}\left(\mathbb{R}^{4}\right)} \leq c\left\|f^{1}\right\|_{L_{2}\left(\mathbb{R}^{4}\right)} .
$$

Using the fact that the solution of problem (6.13) has the form $v=w+\nabla \phi$, where $w$ and $\phi$ are solutions to the problems (6.18) and (6.15) we have that $v \in H^{2 \tilde{s}, \tilde{s}}\left(\mathbb{R}^{4}\right)$ and the following estimate holds

$$
\|v\|_{H^{2 \tilde{s}, \tilde{s}\left(\mathbb{R}^{4}\right)}} \leq\|w\|_{H^{2 \tilde{s}, \tilde{s}\left(\mathbb{R}^{4}\right)}}+\|\nabla \phi\|_{H^{2 \tilde{s}, \tilde{s}\left(\mathbb{R}^{4}\right)}} \leq c\left(\left\|f^{1}\right\|_{L_{2}\left(\mathbb{R}^{4}\right)}+\|\nabla \phi\|_{H^{2,1}\left(\mathbb{R}^{4}\right)}\right),
$$

where

$$
\|\nabla \phi\|_{H^{2,1}\left(\mathbb{R}^{4}\right)} \leq c\left(\|G\|_{H^{1,0}\left(\mathbb{R}^{4}\right)}+\|f\|_{L_{2}\left(\mathbb{R}^{4}\right)}+\lambda\|A\|_{L_{2}\left(\mathbb{R}^{4}\right)}+\|B\|_{L_{2}\left(\mathbb{R}^{4}\right)}\right) .
$$

Using (6.27)-(6.29) we obtain

$$
\begin{aligned}
\|w\|_{H^{2 \tilde{s}, \tilde{s}\left(\mathbb{R}^{4}\right)}}+\|q\|_{\tilde{M}_{2}^{2 \tilde{s}-1}\left(\mathbb{R}^{4}\right)} & \\
& \leq c\left(\|f\|_{L_{2}\left(\mathbb{R}^{4}\right)}+\|G\|_{H^{1,0}\left(\mathbb{R}^{4}\right)}+\lambda\|A\|_{L_{2}\left(\mathbb{R}^{4}\right)}+\|B\|_{L_{2}\left(\mathbb{R}^{4}\right)}\right) .
\end{aligned}
$$

This concludes the proof of the lemma.

From Lemma 6.1, 6.2 and Remark 1-2 we have

Lemma 6.3. Let $f \in L_{2}\left(\mathcal{D}_{T}^{4}\right), G \in H^{1,0}\left(\mathcal{D}_{T}^{4}\right), b_{i} \in H^{2 \tilde{s}-3 / 2, \tilde{s}-3 / 4}\left(\mathbb{R}_{+}^{3}\right) \quad(i=1,2)$, $b_{3} \in H^{2 \tilde{s}-1 / 2, \tilde{s}-1 / 4}\left(\mathbb{R}_{+}^{3}\right)$ and let there exist functions $A, B \in L_{2}\left(\mathcal{D}_{T}^{4}\right)$ such that diam supp $A<\lambda$. Let $\int_{\mathbb{R}_{+}^{3}} p d x=0$ and suppose the following compatibility condition holds

$$
G_{, t}-\operatorname{div} f=\operatorname{div} B+A,
$$

where $\mathcal{D}_{T}^{4}=\mathbb{R}_{+}^{3} \times \mathbb{R}_{+}$. Then system (6.1) has a unique solution $(v, q)$ such as $v \in$ $H^{2 \tilde{s}, \tilde{s}}\left(\mathcal{D}_{T}^{4}\right), q \in \tilde{M}_{2}^{2 \tilde{s}-1}\left(\mathcal{D}_{T}^{4}\right)$ for $\tilde{s} \in(1 / 2,1)$ and the following estimate holds

$$
\begin{aligned}
& \|v\|_{H^{2 \tilde{s}, \tilde{s}\left(\mathcal{D}_{T}^{4}\right)}}+\|q\|_{\tilde{M}_{2}^{2 \tilde{s}-1}\left(\mathcal{D}_{T}^{4}\right)} \leq c\left(\|f\|_{L_{2}\left(\mathcal{D}_{T}^{4}\right)}+\|G\|_{H^{1,0}\left(\mathcal{D}_{T}^{4}\right)}\right. \\
& \left.\quad+\lambda\|A\|_{L_{2}\left(\mathcal{D}_{T}^{4}\right)}+\|B\|_{L_{2}\left(\mathcal{D}_{T}^{4}\right)}+\left\|b_{i}\right\|_{H^{2 \tilde{s}-3 / 2, \tilde{s}-3 / 4}\left(\mathbb{R}_{+}^{3}\right)}+\left\|b_{3}\right\|_{H^{2 \tilde{s}-1 / 2, \tilde{s}-1 / 4}\left(\mathbb{R}_{+}^{3}\right)}\right) .
\end{aligned}
$$


Proof. Let $w_{1}, w_{2}$ be functions such that the domain of $w_{1}$ is $\mathbb{R}^{4}$, the domain of $w_{2}$ is $\mathcal{D}_{T}^{4}$, where $w_{1}$ is the solution to the problem (6.13) and $w_{2}$ is the solution of the system (6.2), for $w_{i}=\left(v_{i}, q_{i}\right) ;(i=1,2)$. One can see that there exist restrictions on functions $v_{1}, q_{1}$ such that

$$
\begin{aligned}
& \left\|\tilde{v}_{1}\right\|_{H^{2 \tilde{s}, \tilde{s}}\left(\mathcal{D}_{T}^{4}\right)} \leq c\left\|v_{1}\right\|_{H^{2 \tilde{s}, \tilde{s}}\left(\mathbb{R}^{4}\right)}, \\
& \left\|\tilde{q}_{1}\right\|_{\tilde{M}_{2}^{2 \tilde{s}-1}\left(\mathcal{D}_{T}^{4}\right)} \leq c\left\|q_{1}\right\|_{\tilde{M}_{2}^{2 \tilde{s}-1}\left(\mathbb{R}^{4}\right)},
\end{aligned}
$$

for $\bar{v}_{1}(x, t)=v_{1}(x, t) \mid(x, t) \in \mathcal{D}_{T}^{4}$ and $\bar{q}_{1}(x, t)=q_{1}(x, t)$ for $(x, t) \in \mathcal{D}_{T}^{4}$. In a similar way we can introduce restrictions of $f, G, A, B$ such that

$$
\begin{array}{ll}
\|f\|_{L_{2}\left(\mathbb{R}^{4}\right)} \leq c\|\tilde{f}\|_{L_{2}\left(\mathcal{D}_{T}^{4}\right)}, & \|G\|_{H^{1,0}\left(\mathbb{R}^{4}\right)} \leq c\|\bar{G}\|_{H^{1,0}\left(\mathcal{D}_{T}^{4}\right)}, \\
\|A\|_{L_{2}\left(\mathbb{R}^{4}\right)} \leq c\|\bar{A}\|_{L_{2}\left(\mathcal{D}_{T}^{4}\right)}, \quad\|B\|_{L_{2}\left(\mathbb{R}^{4}\right)} \leq c\|\bar{B}\|_{L_{2}\left(\mathcal{D}_{T}^{4}\right)} .
\end{array}
$$

If we put $v=\tilde{v}_{1}+v_{2}, q=\tilde{q}_{1}+q_{2}$, It is seen that it is a solution to our problem and the following estimate holds

$$
\begin{aligned}
& \|v\|_{H^{2 \bar{s}, \bar{s}}\left(\mathcal{D}_{T}^{4}\right)}+\|q\|_{\tilde{M}_{2}^{2 \bar{s}-1}\left(\mathcal{D}_{T}^{4}\right)} \leq\left\|\tilde{v}_{1}\right\|_{H^{2 \tilde{s}, \tilde{s}}\left(\mathcal{D}_{T}^{4}\right)}+\left\|\tilde{q}_{1}\right\|_{\tilde{M}_{2}^{\tilde{s}-1}\left(\mathcal{D}_{T}^{4}\right)} \\
& \quad+\left\|v_{2}\right\|_{H^{2 \tilde{s}, \tilde{s}}\left(\mathcal{D}_{T}^{4}\right)}+\left\|q_{2}\right\|_{\tilde{M}^{2 \tilde{s}-1}\left(\mathcal{D}_{T}^{4}\right)} \leq c\left(\left\|v_{1}\right\|_{H^{2 \tilde{s}, \tilde{s}}\left(\mathbb{R}^{4}\right)}\right. \\
& \left.\quad+\left\|q_{1}\right\|_{\bar{M}_{2}^{2 \bar{s}-1}\left(\mathbb{R}^{4}\right)}\right)+\left\|b_{i}\right\|_{H^{2 \bar{s}-3 / 2, \bar{s}-3 / 4}\left(\mathbb{R}_{+}^{3}\right)}+\left\|b_{3}\right\|_{H^{2 \bar{s}-1 / 2, \bar{s}-1 / 4}\left(\mathbb{R}_{+}^{3}\right)},
\end{aligned}
$$

where

$$
\begin{aligned}
\left\|v_{1}\right\|_{H^{2 \bar{s}, \bar{s}\left(\mathbb{R}^{4}\right)}}+\left\|q_{1}\right\|_{\tilde{M}_{2}^{2 \bar{s}-1}\left(\mathbb{R}^{4}\right)} & \leq c\left(\|f\|_{L_{2}\left(\mathbb{R}^{4}\right)}+\|G\|_{H^{1,0}\left(\mathbb{R}^{4}\right)}+\|B\|_{L_{2}\left(\mathbb{R}^{4}\right)}+\lambda\|A\|_{L_{2}\left(\mathbb{R}^{4}\right)}\right. \\
& \left.\leq c\|f\|_{L_{2}\left(\mathcal{D}_{T}^{4}\right)}+\|G\|_{H^{1,0}\left(\mathcal{D}_{T}^{4}\right)}+\lambda\|A\|_{L_{2}\left(\mathcal{D}_{T}^{4}\right)}+\|B\|_{L_{2}\left(\mathcal{D}_{T}^{4}\right)}\right) .
\end{aligned}
$$

From (6.33) and (6.34) we get

$$
\begin{aligned}
& \|v\|_{H^{2 \bar{s}, \bar{s}}\left(\mathcal{D}_{T}^{4}\right)}+\|q\|_{\tilde{M}_{2}^{2 \tilde{s}-1}\left(\mathcal{D}_{T}^{4}\right)} \leq c\left(\|f\|_{L_{2}\left(\mathcal{D}_{T}^{4}\right)}+\|B\|_{L_{2}\left(\mathcal{D}_{T}^{4}\right)}\right. \\
& \left.\quad+\|G\|_{H^{1,0}\left(\mathcal{D}_{T}^{4}\right)}+\lambda\|A\|_{L_{2}\left(D_{T}^{4}\right)}+\left\|b_{i}\right\|_{H^{2 \tilde{s}-3 / 2, \tilde{s}-3 / 4}\left(\mathbb{R}_{+}^{3}\right)}+\left\|b_{3}\right\|_{H^{2 \tilde{s}-1 / 2, \tilde{s}-1 / 4}\left(\mathbb{R}_{+}^{3}\right)}\right) .
\end{aligned}
$$

These considerations finish the proof.

Now using Proposition 2.3, Theorem 2.4 and Lemmas 6.1-6.3 we have the next lemma.

Lemma 6.4. Let $f \in L_{p}\left(\mathcal{D}_{T}^{4}\right), G \in W_{p}^{1,0}\left(\mathcal{D}_{T}^{4}\right), A, B \in L_{p}\left(\mathcal{D}_{T}^{4}\right), \int_{\mathbb{R}^{3}} p d x=0, b_{i} \in$ $\stackrel{\circ}{W}_{p}^{2 s-1-1 / p, s-1 / 2-1 / 2 p}\left(\mathbb{R}_{+}^{3}\right)(i=1,2), b_{3} \in W_{p}^{2 s-1 / p, s-1 / 2 p}\left(R_{+}^{3}\right)$, for $p>\frac{1}{s}, s \in(0,1)$. Assume that the following compatibility condition holds

$$
G_{, t}-\operatorname{div} f=\operatorname{div} B+A .
$$

Then there exists a unique solution to problem $(6.1)$ such that $v \in \stackrel{\circ}{W_{p}^{2 s, s}}\left(\mathcal{D}_{T}^{4}\right), q \in$ $N_{p_{*}}^{\sigma, 0}\left(\mathcal{D}_{T}^{4}\right)$ for some $\sigma \in(0,1)$, and the following estimate holds

$$
\begin{gathered}
\|v\|_{W_{p}^{2 s, s}\left(\mathcal{D}_{T}^{4}\right)}+\|q\|_{N_{p_{*}}^{\sigma, 0}\left(\mathcal{D}_{T}^{4}\right)} \leq c\left(\|f\|_{L_{p}\left(\mathcal{D}_{T}^{4}\right)}+\|G\|_{W_{p}^{1,0}\left(\mathcal{D}_{T}^{4}\right)}\right. \\
\left.+\left\|b_{i}\right\|_{W_{p}^{2 s-1-1 / p, s-1 / 2-1 / 2 p}\left(\mathbb{R}_{+}^{3}\right)}+\left\|b_{3}\right\|_{W_{p}^{2 s-1 / p, s-1 / 2 p}\left(\mathbb{R}_{+}^{3}\right)}+\lambda\|A\|_{L_{p}\left(\mathcal{D}_{T}^{4}\right)}+\|B\|_{L_{p}\left(\mathcal{D}_{T}^{4}\right)}\right)
\end{gathered}
$$

for $N_{p_{*}}^{\sigma, 0}\left(\mathcal{D}_{T}^{4}\right) \equiv \tilde{M}_{p_{*}}^{\sigma, 0}\left(\mathcal{D}_{T}^{4}\right)$.

Proof. We have to consider the interpolations of Banach spaces from the r.h.s. and l.h.s. of our problem. For our considerations we need only describe two types of interpolations, 
first

$$
\left(\stackrel{\circ}{W_{2}^{2 \tilde{s}, \tilde{s}}}\left(\mathcal{D}_{T}^{4}\right), \stackrel{\circ}{W}_{q}^{2,1}\left(\mathcal{D}_{T}^{4}\right)\right)_{\theta, p}=\stackrel{\circ}{W_{p}^{2 s, s}}\left(\mathcal{D}_{T}^{4}\right)
$$

for $\frac{1-\theta}{2}+\frac{\theta}{q}=\frac{1}{p},(1-\theta) \tilde{s}+\theta=s$, and

$$
\left(N_{2}^{2 \tilde{s}-1,0}\left(\mathcal{D}_{T}^{4}\right), N_{q}^{\delta, 0}\left(\mathcal{D}_{T}^{4}\right)\right)_{\theta, p}=N_{p}^{\sigma, 0}\left(\mathcal{D}_{T}^{4}\right)
$$

for $\delta$ which must be found.

Find $\delta \in(0,1\rangle$ such that the following integral inequality holds

$$
\begin{aligned}
& \int_{\mathbb{R}_{+}} \int_{\mathbb{R}_{+}^{3}} \int_{\mathbb{R}_{+}^{3}} \frac{\left|p(x, t)-p\left(x^{\prime}, t\right)\right|^{q}}{\left|x-x^{\prime}\right|^{3+\delta q}} d x d x^{\prime} d t \\
& \quad \leq c\left(\sup _{i=1,2}\left\|b_{i}\right\|_{W_{q}^{1-1 / q, 1 / 2-1 / 2 q}\left(\mathbb{R}_{+}^{3}\right)}+\left\|b_{3}\right\|_{W_{q}^{2-1 / q, 1-1 / 2 q}\left(\mathbb{R}_{+}^{3}\right)}\right) .
\end{aligned}
$$

In order to show such an estimate we need to consider an explicit form of $q$ as a solution of $(6.1)$, where

$$
q=F_{t, x^{\prime}}^{-1}(\hat{q}) ; \quad \hat{q}=\sum_{m=1}^{2} \frac{i \xi m}{\left|\xi^{\prime}\right|} \hat{b}_{m} e^{-\left|\xi^{\prime}\right| x_{3}}+\frac{\left|\xi^{\prime}\right|^{2}+r^{2}}{\left|\xi^{\prime}\right|} \hat{b}_{3} e^{-\left|\xi^{\prime}\right| x_{3}} .
$$

Now one can see that

$$
\begin{aligned}
q(x, t)= & \frac{1}{(2 \pi)^{3 / 2}}\left(\int_{0}^{\infty} e^{s t} \int_{\mathbb{R}^{2}} \frac{\left|\xi^{\prime}\right|^{2}+r^{2}}{\left|\xi^{\prime}\right|} \hat{b}_{3} e^{-\left|\xi^{\prime}\right| x_{3}} d \xi_{0} d \xi^{\prime}\right. \\
& \left.+\sum_{m=1}^{2} \int_{0}^{\infty} e^{s t} \int_{\mathbb{R}^{2}} \frac{i \xi m}{\left|\xi^{\prime}\right|} \hat{b}_{m} e^{-\left|\xi^{\prime}\right| x_{3}} d \xi_{0} d \xi^{\prime}\right),
\end{aligned}
$$

and using the Marcinkiewicz-Mikhlin Theorem [4] we only need to consider

$$
\begin{aligned}
& F_{t, x^{\prime}}^{-1}\left(\left|\xi^{\prime}\right| \hat{b}_{3} e^{-\left|\xi^{\prime}\right| x_{3}}+\hat{b}_{m} e^{-\left|\xi^{\prime}\right| x_{3}}\right) \\
& \quad=F_{t, x^{\prime}}^{-1}\left(\left|\xi^{\prime}\right| e^{-\left|\xi^{\prime}\right| x_{3}}\right) * b_{3}+F_{t, x^{\prime}}^{-1}\left(e^{-\left|\xi^{\prime}\right| x_{3}}\right) * b_{m} \equiv I(t, x)
\end{aligned}
$$

where $I(t, x)=J_{1}+J_{2}$. For simplicity we use that (see [11]) $H_{q}^{\delta, 0}\left(\mathcal{D}_{T}^{4}\right) \subset W_{q}^{\delta, 0}\left(\mathcal{D}_{T}^{4}\right)$, for $\delta \in \mathbb{R}_{+} / \mathbb{Z}_{+} ; q \in\langle 2, \infty)$, and this implies that

$$
\int_{\mathbb{R}_{+}} \int_{\mathbb{R}_{+}^{3}} \int_{\mathbb{R}_{+}^{3}} \frac{\left|q(x, t)-q\left(x^{\prime}, t\right)\right|^{q}}{\left|x-x^{\prime}\right|^{3+\delta q}} d x d x^{\prime} d t \leq c\left(\left\|F_{t, x^{\prime}}^{-1}\left(\left|\xi^{\prime}\right|^{\delta} \hat{q}\right)\right\|_{L_{q}\left(\mathcal{D}_{T}^{4}\right)}+\|\nabla q\|_{L_{q}\left(\mathcal{D}_{T}^{4}\right)}\right) .
$$

Therefore, it is enough to find $\delta$ such that

$$
\left\|F_{t, x^{\prime}}^{-1}\left(\left|\xi^{\prime}\right|^{\delta} \hat{q}\right)\right\|_{L_{q}\left(\mathcal{D}_{T}^{4}\right)} \leq c\left(\left\|b_{i}\right\|_{W_{q}^{1-1 / q, 1 / 2-1 / 2 q}\left(\mathbb{R}_{+}^{3}\right)}+\left\|b_{3}\right\|_{W_{q}^{2-1 / q, 1-1 / 2 q}\left(\mathbb{R}_{+}^{3}\right)}\right) .
$$

So we get

$$
\begin{aligned}
F_{t, x^{\prime}}^{-1}\left(\left|\xi^{\prime}\right|^{\delta} \hat{q}\right)= & \frac{1}{(2 \pi)^{3 / 2}} \int_{0}^{\infty} e^{s t} \int_{\mathbb{R}^{2}} e^{i x^{\prime} \cdot \xi^{\prime}} \frac{\left(\left|\xi^{\prime}\right|^{2}+r^{2}\right)}{\left|\xi^{\prime}\right|}\left|\xi^{\prime}\right|^{\delta} \hat{b}_{3} e^{-\left|\xi^{\prime}\right| x_{3}} d \xi d \xi \\
& +\frac{1}{(2 \pi)^{3 / 2}} \sum_{m=1}^{2} \int_{0}^{\infty} e^{s t} \int_{\mathbb{R}^{2}} e^{i x^{\prime} \cdot \xi^{\prime}} \frac{i \xi m\left|\xi^{\prime}\right|^{\delta}}{\left|\xi^{\prime}\right|} \hat{b}_{m} e^{-\left|\xi^{\prime}\right| x_{3}} d \xi_{0} d \xi^{\prime} .
\end{aligned}
$$

Using the Marcinkiewicz-Mikhlin theorem [4] we only have to estimate the following integrals 


$$
\begin{aligned}
& c \int_{0}^{\infty} e^{s t} \int_{\mathbb{R}^{2}}\left(\left|\xi^{\prime}\right|^{\delta+1} \hat{b}_{3}+\left|\xi^{\prime}\right|^{\delta} \hat{b}_{m}\right) e^{i x^{\prime} \circ \xi^{\prime}} e^{-\left|\xi^{\prime}\right| x_{3}} d \xi_{0} d \xi^{\prime} \\
& \quad \sim c F_{t, x^{\prime}}^{-1}\left(\left|\xi^{\prime}\right| e^{-\left|\xi^{\prime}\right| x_{3}}\right) * F_{t, x^{\prime}}^{-1}\left(\left|\xi^{\prime}\right|^{\delta} \hat{b}_{s}\right)+F_{t, x^{\prime}}^{-1}\left(e^{-\left|\xi^{\prime}\right| x_{3}}\right) * F^{-1}\left(\left|\xi^{\prime}\right|^{\delta} \hat{b}_{1}\right)
\end{aligned}
$$

where

$$
\begin{aligned}
& F_{t, x^{\prime}}^{-1}\left(\left|\xi^{\prime}\right| e^{-\left|\xi^{\prime}\right| x_{3}}\right) * F_{t, x^{\prime}}^{-1}\left(\left|\xi^{\prime}\right|^{\delta} \hat{b}_{3}\right)+F_{t, x^{\prime}}^{-1}\left(e^{-\left|\xi^{\prime}\right| x_{3}}\right) * F^{-1}\left(\left|\xi^{\prime}\right|^{\delta} \hat{b}_{1}\right) \\
& =\int_{\mathbb{R}^{2}} F_{t, x^{\prime}}^{-1}\left(\left|\xi^{\prime}\right| e^{-\left|\xi^{\prime}\right| x_{3}}\right)\left(y^{\prime}, x_{3}\right)\left[F_{t, x^{\prime}}^{-1}\left(\left|\xi^{\prime}\right|^{\delta} \hat{b}_{3}\right)\left(x^{\prime}-y^{\prime}, t\right)-F^{-1}\left(\left|\xi^{\prime}\right|^{\delta} \hat{b}_{3}\right)\left(x^{\prime}, t\right)\right] d y^{\prime} \\
& \quad+\int_{\mathbb{R}^{2}} F_{t, x^{\prime}}^{-1}\left(e^{-\left|\xi^{\prime}\right| x_{3}}\right)\left(y^{\prime}, x_{3}\right)\left[F^{-1}\left(\left|\xi^{\prime}\right|^{\delta} \hat{b}_{1}\right)\left(x^{\prime}-y^{\prime}, t\right)-F^{-1}\left(\left|\xi^{\prime}\right|^{\delta} \hat{b}_{1}\right)\left(x^{\prime}, t\right)\right] d y^{\prime},
\end{aligned}
$$

where

$$
F_{t, x^{\prime}}^{-1}\left(\left|\xi^{\prime}\right|^{\delta} \hat{b}_{k}\right)\left(x^{\prime}-y^{\prime}, t\right)-F_{t}^{-1}\left(\left|\xi^{\prime}\right|^{\delta} \hat{b}_{k}\right)\left(x^{\prime}, t\right)=\Delta_{(-y)} F_{t, x^{\prime}}^{-1}\left(\left|\xi^{\prime}\right|^{\delta} \hat{b}_{k}\right)\left(x^{\prime}, t\right)
$$

for $\left(\Delta_{(a)} T\right)(x) \equiv T(x+a)-T(x)$. One can also calculate that

$$
F_{t, x^{\prime}}^{-1}\left(e^{-\left|\xi^{\prime}\right| x_{3}}\right)=\frac{x_{3}}{\left(x_{1}^{2}+x_{2}^{2}+x_{3}^{2}\right)^{3 / 2}} \equiv K,
$$

and

$$
F_{t, x^{\prime}}^{-1}\left(\xi_{j} e^{-\left|\xi^{\prime}\right| x_{3}}\right)=\partial_{x_{j}} K=\frac{x_{3} x_{j}}{\left(x_{1}^{2}+x_{2}^{2}+x_{3}^{2}\right)^{5 / 2}}, \quad(j=1,2) .
$$

Because $K$ as a function of $x_{j}$, satisfies the following equality $K\left(-x_{j}\right)=K\left(x_{j}\right)$, then $\partial_{x_{j}} K\left(-x_{j}\right)=-\partial_{x_{j}} K\left(x_{j}\right)$, and this implies that $\int \partial_{x_{j}} K d x^{\prime}=0$, for $x^{\prime}=\left(x_{1}, x_{2}\right)$.

We can start now to estimate the first integral in the $L_{q}$-norm. Let

$$
A_{1}=\int_{\mathbb{R}^{2}} d y^{\prime} \partial_{, y_{j}} K\left(y^{\prime}, x_{3}\right)\left[\Delta_{(-y)} F_{t, x^{\prime}}^{-1}\left(\left|\xi^{\prime}\right|^{\delta} \hat{b}_{3}\right)\right]\left(x^{\prime}, t\right) .
$$

Then we have

$$
\left\|A_{1}\right\|_{L_{q}\left(R_{+}^{3}\right)}=\left(\int_{\mathbb{R}_{+}^{3}}\left|A_{1}\right|^{q} d x^{\prime} d t\right)^{1 / q} \leq c \int_{\mathbb{R}^{2}}\left|\partial_{, y_{j}} K\left(y^{\prime}, x_{3}\right)\right| R\left(y^{\prime}\right) d y^{\prime},
$$

where

$$
R\left(y^{\prime}\right) \equiv\left\|\left[\Delta_{\left(-y^{\prime}\right)} F_{t, x^{\prime}}^{-1}\left(\left|\xi^{\prime}\right|^{\delta} \hat{b}_{3}\right)\right]\left(x^{\prime}, t\right)\right\|_{L_{q}\left(\mathbb{R}_{+} \times \mathbb{R}_{x^{\prime}}^{2}\right)} .
$$

Using the definition of $F_{t, x^{\prime}}^{-1}$ one can see that

$$
R\left(y^{\prime}\right) \sim\left(\int_{\mathbb{R}_{+}} \int_{\mathbb{R}_{+}^{2}} \frac{\left|b_{3}\left(x^{\prime}-y^{\prime}, t\right)-b_{3}\left(x^{\prime}, t\right)\right|^{q}}{\left|y^{\prime}\right|^{\delta q}} d x^{\prime} d t\right)^{1 / q} .
$$

Now we estimate $\left\|A_{1}\right\|_{L_{q}\left(\mathcal{D}_{T}^{4}\right)}$. One can see that

$$
\begin{aligned}
\left\|A_{1}\right\|_{L_{q}\left(\mathcal{D}_{T}^{4}\right)}^{q} & \leq c \int_{\mathbb{R}_{+}} d x_{3} \int_{\mathbb{R}^{2}} d y^{\prime} \frac{\left|y_{j}\right|^{q} x_{3}}{\left(y^{\prime 2}+x_{3}^{2}\right)^{3 / 2+q}} R^{q}\left(y^{\prime}\right)\left(\int_{\mathbb{R}^{2}} \frac{x_{3} d y^{\prime}}{\left(\left|y^{\prime}\right|^{2}+x_{3}\right)^{3 / 2}}\right)^{q / q^{*}} \\
& \leq c \int_{0}^{\infty} d x_{3} \int_{\mathbb{R}^{2}} d y^{\prime} \frac{y_{j}^{q} x_{3}}{\left(\left|y^{\prime}\right|^{2}+x_{3}^{2}\right)^{q+3 / 2}}\left|y^{\prime}\right|^{\alpha} \frac{R^{q}\left(y^{\prime}\right)}{\left|y^{\prime}\right|^{\alpha}}
\end{aligned}
$$

Let us examine the integral

$$
\int_{0}^{\infty} d x_{3} \frac{x_{3} y_{j}^{q}\left|y^{\prime}\right|^{\alpha}}{\left(\left|y^{\prime}\right|^{2}+x_{3}^{2}\right)^{q+3 / 2}}
$$


Taking $w=\frac{x_{3}}{\left|y^{\prime}\right|}$, we get

$$
\int_{0}^{\infty} d w \frac{w\left|y^{\prime}\right|^{q+2+\alpha}}{\left|y^{\prime}\right|^{2 q+3}\left(1+w^{2}\right)^{q+3 / 2}} .
$$

Now we need to show independence of $\left|y^{\prime}\right|$. This implies that $\alpha=q+1$. Using this we have the following inequality

$$
\left\|A_{1}\right\|_{L_{q}\left(\mathcal{D}_{T}^{4}\right)}^{q} \leq c \int_{\mathbb{R}_{+}} \int_{\mathbb{R}_{+}^{2}} \int_{\mathbb{R}_{+}^{2}} \frac{\left|b_{3}\left(t, x^{\prime}-y^{\prime}\right)-b_{3}\left(t, x^{\prime}\right)\right|^{q}}{\left|y^{\prime}\right|^{\alpha+\delta q}} d x^{\prime} d y^{\prime} d t .
$$

From the assumption we have that $b_{3} \in W_{q}^{2-1 / q, 1-1 / 2 q}\left(\mathbb{R}_{+}^{3}\right)$, where

$$
\left\|b_{3}\right\|_{W_{q}^{2-1 / q, 1-1 / 2 q}\left(R_{+}^{3}\right)} \sim \int_{\mathbb{R}_{+}}\left\|b_{3}\right\|_{W_{q}^{2-1 / q}\left(\mathbb{R}^{2}\right)} d \tau+\int_{\mathbb{R}^{2}}\left\|b_{3}\right\|_{W_{q}^{1-1 / q}\left(\mathbb{R}_{+}\right)} d x^{\prime}
$$

and

$$
\begin{aligned}
\int_{\mathbb{R}_{+}} & \left\|b_{3}\right\|_{W_{q}^{2-1 / q}\left(\mathbb{R}^{2}\right)} \sim \int_{\mathbb{R}_{+}}\left(\int_{\mathbb{R}^{2}}\left|b_{3}\right|^{q} d x^{\prime}+\int_{\mathbb{R}^{2}}\left|D_{x^{\prime}} b_{3}\right|^{q} d x^{\prime}\right. \\
& +\int_{\mathbb{R}^{2}} \int_{\mathbb{R}^{2}} \frac{\left|b_{3}\left(x^{\prime}-y^{\prime}, t\right)-b\left(x^{\prime}, t\right)\right|^{q}}{\left|y^{\prime}\right|^{2+q(1-1 / q)}} d x^{\prime} d y^{\prime} \\
& \left.+\int_{\mathbb{R}^{2}} \int_{\mathbb{R}^{2}} \frac{\left|D_{y^{\prime}} b_{3}\left(x^{\prime}-y^{\prime}, t\right)-D_{x^{\prime}} b\left(x^{\prime}, t\right)\right|^{q}}{\left|y^{\prime}\right|^{2+q(1-1 / q)}} d x^{\prime} d y^{\prime}\right) d t
\end{aligned}
$$

Using that there exists $c>0$ such that

$$
\left|b_{3}\left(x^{\prime}-y^{\prime}, t\right)-b_{3}\left(x^{\prime}, t\right)\right|^{q} \leq c\left|D_{y^{\prime}} b_{3}\left(x^{\prime}-y^{\prime}, t\right)-D_{x^{\prime}} b_{3}\left(x^{\prime}, t\right)\right|^{q}\left|y^{\prime}\right|^{q},
$$

we get the following inequality

$$
\begin{aligned}
& \int_{\mathbb{R}_{+}} \int_{\mathbb{R}_{+}^{2}} \int_{\mathbb{R}_{+}^{2}} \frac{\left|b_{3}\left(x^{\prime}-y^{\prime}, t\right)-b_{3}\left(x^{\prime}, t\right)\right|^{q}}{\left|y^{\prime}\right|^{\alpha+\delta q}} d x^{\prime} d y^{\prime} d t \\
& \quad \leq c \int_{\mathbb{R}_{+}} \int_{\mathbb{R}_{+}^{2}} \int_{\mathbb{R}_{+}^{2}} \frac{\left|b_{3}\left(x^{\prime}-y^{\prime}, t\right)-b_{3}\left(x^{\prime}, t\right)\right|^{q}}{\left|y^{\prime}\right|^{2+q+q(1-1 / q)}} d x^{\prime} d y^{\prime} d t \\
& \quad \leq c\left\|b_{3}\right\|_{W_{q}^{2-1 / q, 1-1 / 2 q}\left(\mathbb{R}_{+}^{3}\right)},
\end{aligned}
$$

which holds for $\alpha+\delta q \leq 2+q+q(1-1 / q)$, where $\alpha=q+1$. This implies that inequality (6.50) holds for $\delta \leq 1$. Next we have to consider the second integral $A_{2}$ where

$$
A_{2}=\int_{\mathbb{R}^{2}} d y^{\prime} K\left(y^{\prime}, x_{3}\right)\left[\Delta_{\left(-y^{\prime}\right)} F_{t, x^{\prime}}^{-1}\left(\left|\xi^{\prime}\right|^{\delta} \hat{b}_{m}\right)\right]\left(x^{\prime}, t\right),
$$

for $m=1,2$. Using (6.45) we have that

$$
A_{2}=F_{t, x^{\prime}}^{-1}\left(e^{-\left|\xi^{\prime}\right| x_{3}}\right) * F_{t, x^{\prime}}^{-1}\left(\left|\xi^{\prime}\right|^{\delta} \hat{b}_{m}\right)=F_{t, x^{\prime}}^{-1}\left(\left|\xi^{\prime}\right|^{\delta} e^{-\left|\xi^{\prime}\right| x_{3}}\right) * b_{m},
$$

and

$$
A_{1}=F_{t, x^{\prime}}^{-1}\left(\left|\xi^{\prime}\right|^{\delta} e^{-\left|\xi^{\prime}\right| x_{3}}\right) * F^{-1}\left(\left|\xi^{\prime}\right| \hat{b}_{3}\right)=F_{t, x^{\prime}}^{-1}\left(\left|\xi^{\prime}\right|^{\delta} e^{-\left|\xi^{\prime}\right| x_{3}}\right) *\left(D_{x^{\prime}} b_{3}\right),
$$

where we exploited properties of $F_{t, x^{\prime}}^{-1}$ and of the convolution. From (6.39) we have that $D_{x^{\prime}} b_{3} \in W_{q}^{1-1 / q, 1 / 2-1 / 2 q}\left(\mathbb{R}_{+}^{3}\right)$, and (6.52) with $\delta \leq 1$ yields

$$
\left\|A_{1}\right\|_{L_{q}\left(\mathcal{D}_{T}^{4}\right)} \leq c\left\|D_{x^{\prime}} b_{3}\right\|_{W_{q}^{1-1 / q, 1 / 2-1 / 2 q}\left(\mathbb{R}_{+}^{3}\right)} .
$$


From (6.53)-(6.56) we get an estimate

$$
\left\|A_{2}\right\|_{L_{q}\left(\mathcal{D}_{T}^{4}\right)} \leq c\left\|b_{m}\right\|_{W_{q}^{1-1 / q, 1 / 2-1 / 2 q}\left(\mathbb{R}_{+}^{3}\right)},
$$

for $\delta \leq 1$.

All these considerations imply that if $\delta=1$ (6.39) also holds. Now we can consider the following interpolation space $N_{p}^{\sigma, 0}\left(\mathcal{D}_{T}^{4}\right)$, where

$$
N_{p_{*}}^{\sigma, 0}\left(\mathcal{D}_{T}^{4}\right)=\left(N_{2}^{2 \tilde{s}-1,0}\left(\mathcal{D}_{T}^{4}\right), N_{q}^{1,0}\left(\mathcal{D}_{T}^{4}\right)\right)_{\theta, p}
$$

for some $\sigma$ and $p_{*}$, and

$$
N_{2}^{2 \tilde{s}-1,0}\left(\mathcal{D}_{T}^{4}\right) \cap N_{q}^{1,0}\left(\mathcal{D}_{T}^{4}\right) \subset N_{p_{*}}^{\sigma, 0}\left(\mathcal{D}_{T}^{4}\right) \subset N_{2}^{2 \tilde{s}-1,0}\left(\mathcal{D}_{T}^{4}\right)+N_{q}^{1,0}\left(\mathcal{D}_{T}^{4}\right) .
$$

Using that

$$
\stackrel{\circ}{W_{p}^{2 s, s}}\left(\mathcal{D}_{T}^{4}\right)=\left(\stackrel{\circ}{W_{2}^{2 \tilde{s}, \tilde{s}}}\left(\mathcal{D}_{T}^{4}\right) ; \stackrel{\circ}{W_{q}^{2,1}}\left(\mathcal{D}_{T}^{4}\right)\right)_{\theta, p}
$$

and (6.58) and considerations similar to those from the proof of Lemma 3.2 we show Lemma 6.4. These considerations end the proof of the lemma.

Using now Lemmas 6.1-6.4 and considerations similar to those in Chapters 4 and 5 we finish the proof of Theorem 2.

Acknowledgments. The author is greatly indebted to Prof. Wojciech Zajączkowski for very fruitful discussions during the preparation of this paper, and for his help in education in mathematics.

\section{References}

[1] W. Alame, On the existence of solutions for the nonstationary Stokes system with slip boundary conditions, Appl. Math. 32 (2005), 195-223.

[2] H. Amann, On the strong solvability of the Navier-Stokes equations, J. Math. Fluid Mech. 2 (2000), 16-98.

[3] O. V. Besov, V. P. Il'in and S. M. Nikol'skiŭ, Integral Representations of Functions and Imbedding Theorems, Nauka, Moscow, 1975 (in Russian).

[4] J. Marcinkiewicz, Sur les multiplicateurs des séries de Fourier, Studia Math. 8 (1939), 78-91.

[5] P. B. Mucha and W. Zajączkowski, On the existence for the Cauchy-Neumann problem for the Stokes system in $L_{p}$-framework, Studia Math. 143 (2000), 76-101.

[6] J. Peetre, A Theory of Interpolation of Normed Space, Notes Universidade de Brasilia 1963 [Notas de matematica 39 (1968)].

[7] J. Peetre, Interpolation i abstracta wm, Lecture Notes, Lund, 1966.

[8] J. Peetre, A new approach in interpolation spaces, Studia Math. 34 (1970), 23-42.

[9] J. Peetre, Banach couples, I. Elementary theory techn. report, Lund, 1971.

[10] Yu. Petunin, Interpolation between factor spaces, Ukrain. Mat. Zh. 23 (1971), 157-167 (in Russian).

[11] H. Triebel, Interpolation Theory, Function Spaces, Differential Operators, Deutscher Verlag der Wissenschaften, Berlin, 1978. 